\title{
Magnetic resonance imaging of the wrist and hand
}

\author{
Ravi Vassa ${ }^{\mathrm{E}, \mathrm{F}}$, Ankur Garg ${ }^{\mathrm{E}, \mathrm{F}}$, Imran Muhammad Omar ${ }^{\mathrm{E}, \mathrm{F}}$ \\ Department of Radiology, Northwestern University Feinberg School of Medicine, USA
}

\section{Abstract}

The anatomy of the wrist and hand is complex and contains numerous small structures. Magnetic resonance imaging (MRI) is often an ideal imaging modality in the assessment of various traumatic and pathologic conditions of this region, and it is frequently performed after initial radiographs. In this manuscript we describe the normal anatomy, imaging techniques, and MRI findings of various traumatic and pathologic conditions of the wrist and hand including occult fractures, osteonecrosis, ligamentous and tendon injuries, and entrapment neuropathies.

Key words: ligament, MRI, tendon, wrist, osteonecrosis, hand.

\section{Introduction}

The wrist and hand are composed of numerous small structures with complex morphologies and anatomic variations, which makes diagnostic imaging challenging. Magnetic resonance imaging (MRI) has high spatial and contrast resolution, and can characterise bone and soft tissue without using ionising radiation, making it an ideal imaging modality to assess pathologic conditions affecting joints. In our practice, the most common indications for imaging include the following: detecting radiographically occult osseous injuries (Figure 1), assessment of the triangular fibrocartilage complex (TFCC) and intrinsic ligaments of the wrist, ligament tears involving the fingers, and flexor and extensor tendon injuries. Additionally, MRI is very helpful in characterising palpable abnormalities, such as ganglion cysts, soft tissue masses, and osseous excrescences [1,2], as well as assessing for causes of peripheral neuropathies of the median and ulnar nerves within the wrist and hand (Figure 2) [3].

\section{Magnetic resonance imaging techniques}

MRI of the hand and wrist requires optimising imaging parameters, including small field of view (often $8 \mathrm{~cm}$ for the wrist), slice thickness (often 3-4 mm), band width, repetition time (TR), and echo time (TE), in as short a time as possible to avoid patient discomfort and fatigue. Most MRI studies are currently performed at higher field strength (ideally $3 \mathrm{~T}$ ), which significantly improves spatial and contrast resolution to enable the assessment of joint internal derangement [4]. Current protocols utilise 2-D fast spin echo imaging in a combination of chemicallyselective fat-suppressed (FS), fluid-sensitive sequences, such as T2-weighted (T2W) or proton density (PD) sequences in three orthogonal planes, to look for marrow and soft tissue oedema (Figure 3), in addition to a coronal T1-weighted (T1W) non-fat suppressed (NFS) sequence, which is often used to detect osseous alignment, fracture lines, and marrow replacement [5]. Many institutions also perform thin section gradient echo sequences to rapidly obtain thin section, high spatial resolution imaging to better assess the TFCC and articular cartilage (Figure 4) [6]. However, these sequences often suffer from poor contrast resolution, which may make articular cartilage and TFCC injuries less conspicuous [7]. Newer three-dimensional spin echo techniques allow thin section imaging with isotropic voxels and no interslice gaps. This decreases volume averaging and allows the generation of multiplanar reformatted imaging in any desired plane [8].

Patients should be placed prone with the arm extended over head in the "Superman" position to put the wrist

\section{Correspondence address:}

Dr. Imran Muhammad Omar, Department of Radiology, Department of Radiology Northwestern University Feinberg School of Medicine, USA, e-mail: iomar@nm.org Authors' contribution:

A Study design · B Data collection · C Statistical analysis · D Data interpretation · E Manuscript preparation · F Literature search · G Funds collection 


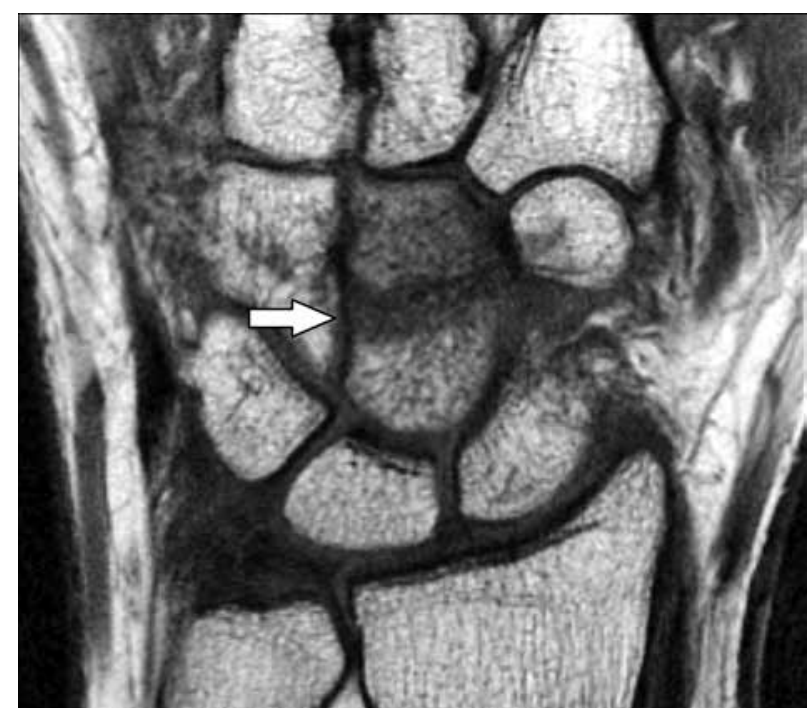

Figure 1. Radiographically occult capitate fracture seen on magnetic resonance (MR) imaging. 22-year-old female with traumatic left wrist injury. Coronal T1W non-fat suppressed MR image of the wrist shows a transverse fracture of the capitate waist (arrow) that was not visible radiographically (not shown)

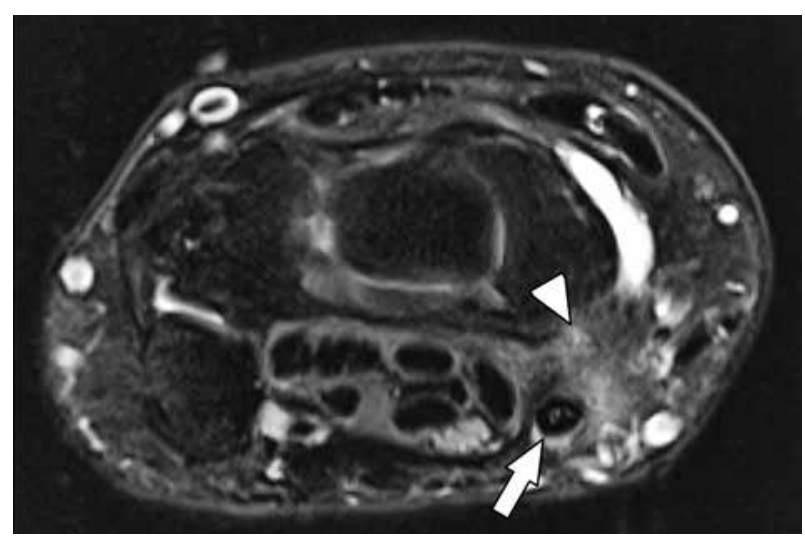

Figure 3. Tendinosis and tenosynovitis of the flexor carpi radialis tendon. 52-year-old female with 8 months of radial/volar left wrist pain after a fall. $2 \mathrm{D}$ fast spin echo $\mathrm{T} 2 \mathrm{~W}$ fat-suppressed magnetic resonance image at the level of the mid carpus shows mild flexor carpi radialis tendinosis (arrow), mild tendon sheath distension and ill-defined soft tissue oedema deep to the tendon consistent with tenosynovitis (arrowhead)

as close to the isocentre of the magnetic field as possible, in order to obtain the highest signal-to-noise ratio (SNR) and most homogeneous signal. However, this can be uncomfortable for many patients, and some patients may be unable to complete the study in this position $[1,9]$. As a result, many institutions have elected to perform imaging with the patient supine and the arm at the side, which brings the hand away from the isocentre of the magnetic field and may lead to decreased signal throughout the image with poor and inhomogeneous fat suppression. If incomplete or heterogeneous fat saturation impacts diagnostic quality, chemically selective fat suppression techniques can be switched to a short tau inversion recovery (STIR) sequence. STIR imaging produces homogeneous fat suppression throughout the image. However, there is generally poorer SNR and spatial resolution compared to

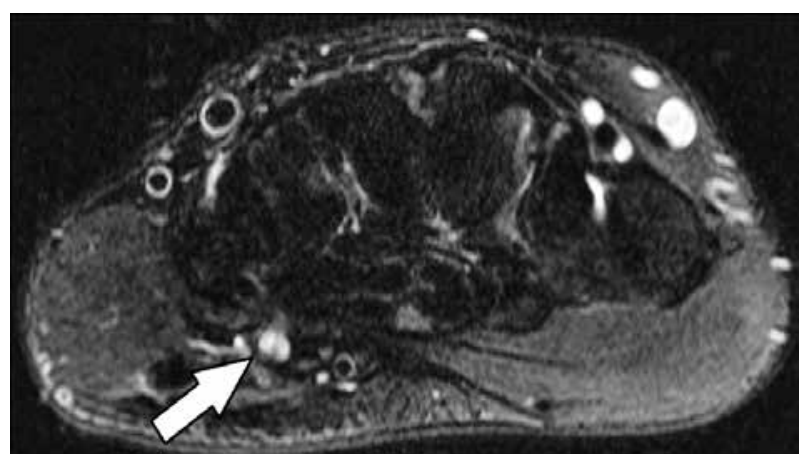

Figure 2. Detection of peripheral neuropathy on magnetic resonance (MR) imaging. 31-year-old male with one-year history of ulnar-sided wrist pain after an injury. Axial T2W fat-suppressed MR image at the level of the hook of the hamate shows oedema of the deep branch of the ulnar nerve as it courses over the hook of the hamate (arrow), which could account for the patient's symptoms

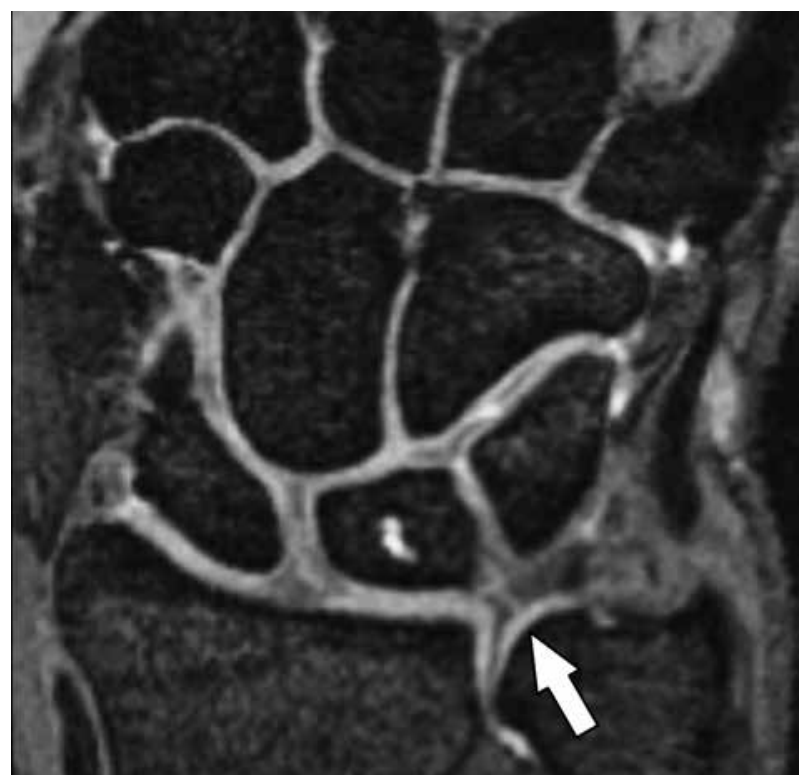

Figure 4. 3D gradient echo (GRE) imaging showing a central triangular fibrocartilage disc (TFC) perforation. Coronal T2W fat-suppressed GRE magnetic resonance image with thin section clearly shows a linear central perforation in the TFC (arrow)

chemically selective FS spin-echo techniques. Another described position, the "Prayer configuration" position, can be used with the patient on his or her side and the elbow flexed, thereby bringing the wrist near the face, closer to the magnet's isocentre [10].

Intravenous gadolinium contrast is not needed to characterise internal derangements of the wrist and hand. Most commonly it is useful in the setting of palpable soft tissue abnormalities to differentiate solid from cystic lesions, and to detect areas of active synovitis in the setting of inflammatory arthropathy, either in the initial diagnosis of an inflammatory arthropathy or to follow therapeutic response $[11,12]$. Occasionally, contrast can be helpful to detect areas of granulation tissue or hyperaemia associated with soft tissue injuries that may be less conspicuous on routine, non-contrast imaging. At our institution, the most com- 


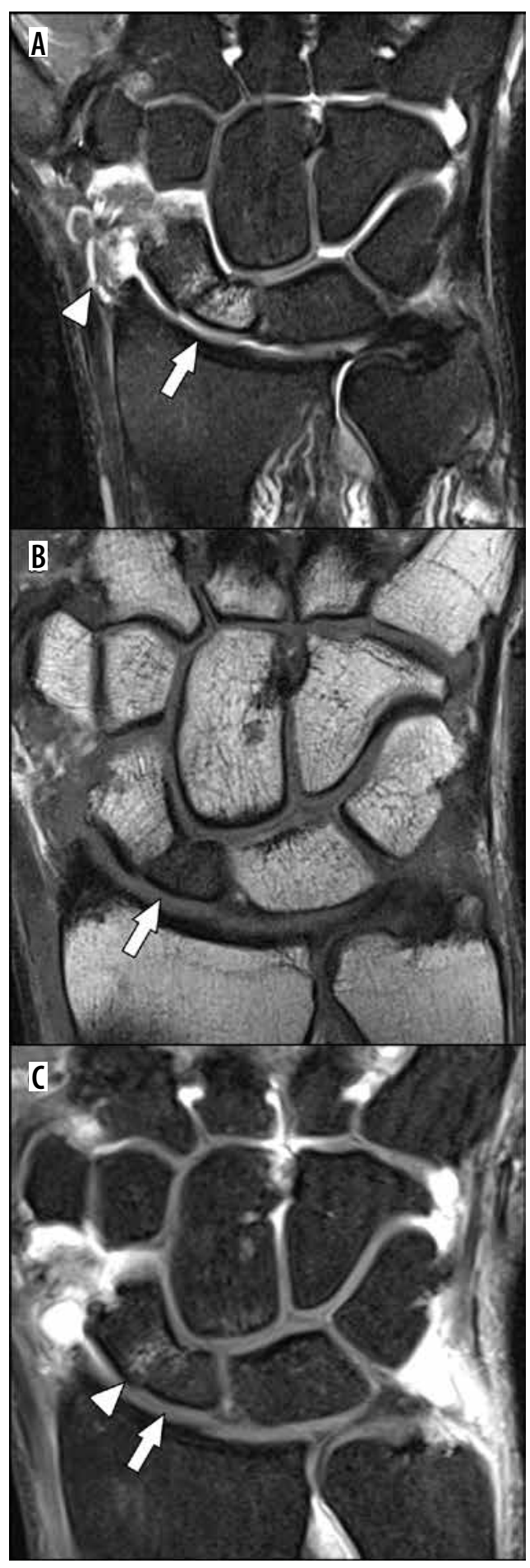

Figure 5. Use of intravenous contrast to determine bone viability following carpal fracture. 24-year-old male with mildly displaced scaphoid waist fracture nonunion, and persistent pain. A) Coronal T2W fat-suppressed (FS) magnetic resonance (MR) image of the wrist shows reciprocal bone marrow oedema within the scaphoid centred along the fracture that extends throughout the proximal pole (arrow). Additionally, the radial collateral ligament is oedematous and thickened, consistent with partial tearing (arrowhead). B) Coronal T1W non-fat suppressed MR image of the same region shows replacement of the normal fat in the scaphoid proximal pole (arrow). These findings are not specific for osteonecrosis but may suggest it. C) Coronal post contrast T1W FS MR image demonstrates no enhancement of the proximal scaphoid pole, indicating osteonecrosis (arrow). There is mild enhancement of the articulating margin of the distal pole fragment (arrowhead) that was felt to represent fibrovascular ingrowth

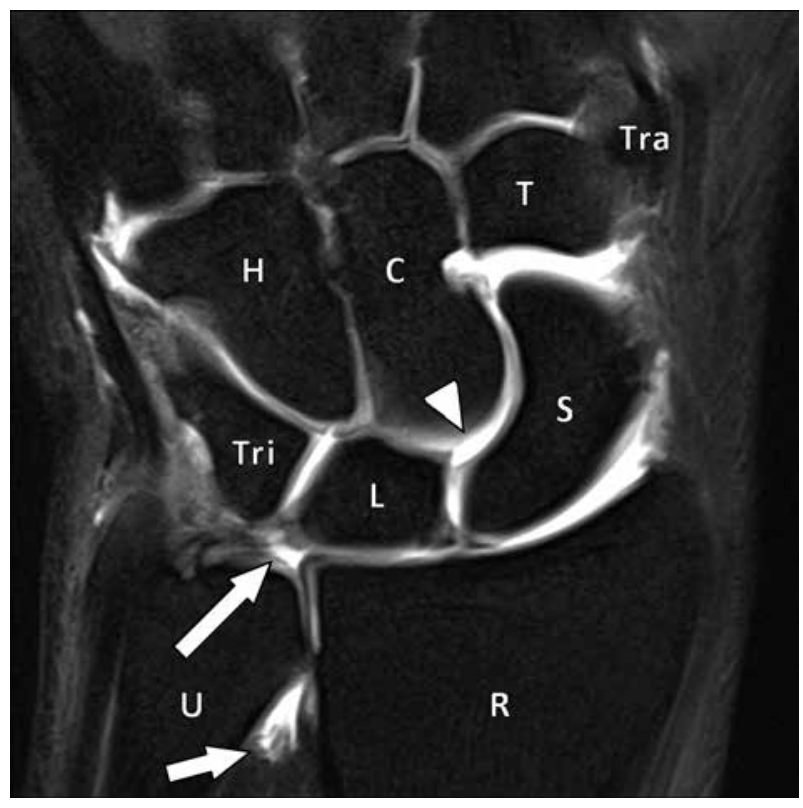

Figure 6. Single compartment magnetic resonance (MR) arthrography in a 31-year-old male with ulnar-sided wrist pain. Coronal T1W fat-suppressed MR image of the carpus following radioscaphoid injection of dilute gadolinium demonstrates contrast extending into distal radioulnar joint (shorter arrow), indicating a full-thickness central triangular fibrocartilage disc (TFC) tear (longer arrow), as well as the mid carpal joint (arrowhead), indicating either an scapholunate (SLL) or lunotriquetral tear. At surgery, there were a central TFC tear and an SLL tear. The carpal bones are also labelled ( $U$ - ulna, $\mathrm{R}$ - radius, Tri - triquetrum, $\mathrm{L}$ - lunate, $\mathrm{S}$ - scaphoid, $\mathrm{H}$ - hamate, $\mathrm{C}$ - capitate, $\mathrm{T}$ - trapezoid, $\mathrm{Tra}$ - trapezium)

mon indication for intravenous contrast is to better characterise suspected infections. Contrast can distinguish viable from non-viable soft tissue, such as helping to discriminate developing abscesses (which exhibit peripheral enhancement) from phlegmon (which demonstrates more diffuse enhancement) [13]. Furthermore, some institutions have used contrast to assess whether bones, such as the scaphoid following a proximal pole or a waist fracture, demonstrate signs of osteonecrosis (Figure 5). While some authors have advocated performing these studies with dynamic postcontrast imaging to determine the rate of enhancement and assess for potential ischaemia, there is debate about whether dynamic imaging is better than routine MRI [14-16].

Magnetic resonance arthrography (MRA) has been shown to improve sensitivity and diagnostic accuracy in detecting injuries to the TFCC and intrinsic wrist ligaments by increasing the contrast resolution between normal structures and regions of pathology $[17,18]$. Two methods of MRA have been described: direct, in which dilute gadolinium contrast is percutaneously injected into the target joint (Figure 6), and indirect, in which contrast is injected intravenously, allowed to circulate systemically (Figure 7), and recruited to a specific joint via exercise-induced hyperaemia [5]. Historically, direct wrist arthrography used a two- or three-compartment approach, whereby contrast was injected into multiple compartments of the wrist, and imaging (MRI or radiography) was performed to determine whether there was abnormal communication between 


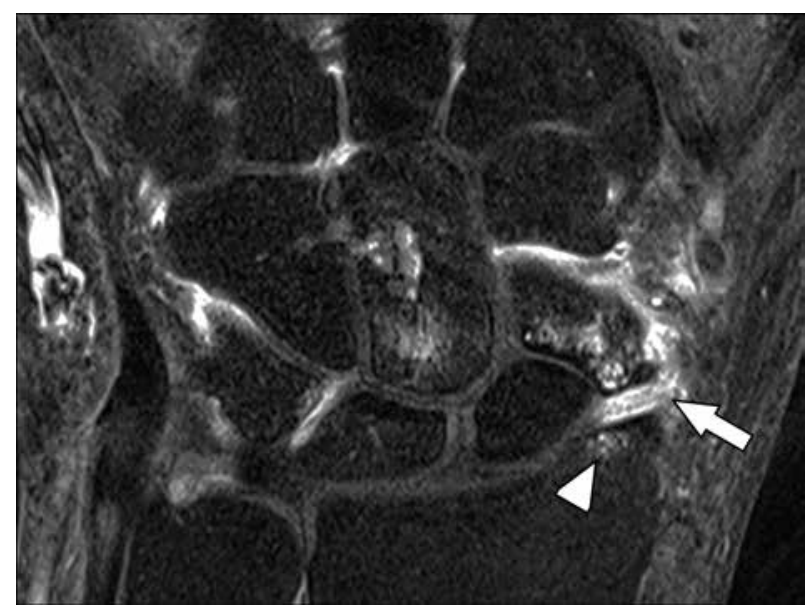

Figure 7. Indirect magnetic resonance arthrography showing localised synovitis in an area of symptomatic impingement in a 46-year-old female with chronic scaphoid fracture with nonunion and persistent radial-sided wrist pain. Coronal T1W fat-suppressed magnetic resonance image of the wrist depicts a non-united scaphoid waist fracture with enhancing bone marrow oedema in the distal pole about the fracture site. The tip of the radial styloid process is remodelled and abuts against the distal scaphoid pole (arrowhead). There is localised enhancement related to focal synovitis (arrow), and the patient was diagnosed with radial styloid impingement

the compartments that indicated a tear in the intervening supporting structures [19]. More recently, many centres have favoured a single compartment approach into the radiocarpal joint because the multiple compartment approach can cause discomfort to the patient and result in false negative studies [20], and the single compartment injection should increase the intra-articular pressure and drive contrast into an adjacent compartment if there is an abnormal communication $[21,22]$. In indirect arthrography, the synovium begins to excrete gadolinium at about 10-11 minutes, and postcontrast imaging is usually performed at about 25-30 minutes following the injection to allow enough gadolinium to enter the joint $[23,24]$.

Some institutions have advocated placing traction on the wrist during the MRA with finger traps connected to a pulley and weight system. Although this technique is not widespread, it may improve visualisation of wrist cartilage, and intrinsic and extrinsic wrist ligament pathology. However, it may not be very helpful in improving characterisation of TFCC injuries [25].

In addition to the small risks of infection, bleeding and injury to local structures, direct MRA does have some pitfalls and drawbacks that should be considered. First, it is invasive and can cause a reactive synovitis that can produce pain for several days following the injection. Furthermore, increasing the intra-articular pressure can cause extravasation of the dilute gadolinium, either along the needle tract or through weaker areas of the joint capsule, which can either obscure or mimic pathology. Finally, it results in greater economic cost and takes more time to perform [22].

Indirect MRA is much less common than direct MRA because it does not increase intra-articular pressure, which may prevent adequate visualisation of pathology. Further-

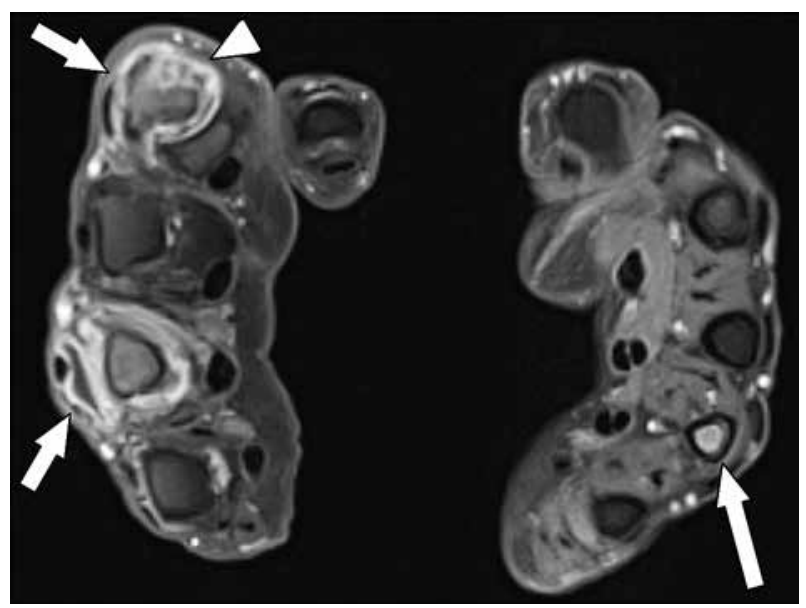

Figure 8. Post-contrast magnetic resonance imaging (MRI) of the hands for inflammatory arthritis. MRI of both hands was performed on a 73-year-old female with history of rheumatoid arthritis. The hands were imaged with the palms facing one another. Axial T1W fat-suppressed post-contrast MRI shows intense synovial enhancement of the right second and fourth metacarpophalangeal (MCP) joints (shorter arrows) with marginal erosions of the second metacarpal head (arrowhead). There is additional, mild distal fourth metacarpal bone marrow enhancement (longer arrow) on the contralateral left hand. However, no synovial inflammation at the left MCP joints was seen

more, the gadolinium contrast is systemic, and synovial enhancement of two adjacent wrist compartments can mimic abnormal communication, even if the intervening soft tissue support structures are intact. This technique provides functional information by detecting areas of active synovitis, which may contribute to patient symptoms (Figure 7).

\section{Magnetic resonance imaging of the hand}

MRI of the entire hand is relatively less common since the larger field of view can reduce spatial resolution and impede ability to detect internal derangement. At our institution, the most common indication is inflammatory arthritis, in which we usually place the patient in the Superman position. If both hands require assessment, then both arms would be extended overhead with the hands placed together and the palms facing one another so that the opposing corresponding joints can be compared on the same images (Figure 8). Imaging is performed with a knee coil or a flexible surface coil. Some institutions may separate the hands slightly with a block or towel, while others place the hands with the palms touching. A larger field-of-view MRI of the entire hand can also be used to assess conditions that can affect both the hand and the wrist, such as suspected infection, and for which assessment of small stabilising structures, such as the collateral ligaments or tendon pulleys, is less important [26].

\section{Magnetic resonance imaging of the lesser fingers}

When possible, MRI of the fingers should be performed on $3 \mathrm{~T}$ systems to obtain superior spatial resolution, and the fingers should be placed in a dedicated 
hand/wrist coil. Similar to imaging of the wrist, many routine MRI protocols contain a combination of fatsuppressed, fluid-sensitive sequences and NFS T1W and PD sequences. Long-axis (sagittal and coronal) imaging of a finger should be planned from axial images of the individual finger rather than assuming the orientation is identical to that of the hand. Furthermore, many institutions include at least one adjacent, asymptomatic finger to serve as an internal control for the appearance of these structures [27]. Some authors have suggested that semidynamic stress imaging of the fingers with finger flexion may be helpful to detect flexor pulley injuries with palmar displacement of the tendon (bowstringing), which can be occult when the fingers are extended (Figure 9) [28].

\section{Magnetic resonance imaging of the thumb}

Many of the technical considerations used in imaging the lesser fingers are common to the thumb. However, the thumb is generally extended, rotated, and abducted with respect to the hand and lesser fingers. As a result, sagittal and coronal imaging should be oriented with respect to the thumb to optimise visualisation of the ligaments and tendons. To do this the coronal sequence should be planned from the axial images through the first metacarpal head and metacarpophalangeal (MCP) joint [29]. This plane best visualises the collateral ligaments. The sagittal plane is $90^{\circ}$ perpendicular to the coronal plane based on axial imaging, and is best used to examine the tendons, palmar plate, and some flexor pulley injuries.

\section{Magnetic resonance imaging of the wrist}

\section{Anatomy}

\section{Bones}

The wrist is perhaps the most complex joint in the body and is affected by a wide spectrum of disorders. It is composed of the distal radius and ulna, carpal bones, and five metacarpal bases. Eight carpal bones articulate to form the carpus, which can be divided into two horizontal rows (Figure 6) [26]. The proximal carpal row is composed of the scaphoid, lunate, and triquetrum. The pisiform is considered a sesamoid bone and not part of the proximal carpal row [30]. However, it can indirectly affect the triquetrum. The distal carpal row is composed of the trapezium, trapezoid, capitate, and hamate. These bones form an arch that supports the metacarpals and is considered more stable than the proximal row.

The carpal vascular anatomy is considered atypical because the blood vessels often enter the bones distally. This pattern of blood supply places the proximal segments of the bones at risk for osteonecrosis or nonunion after fracture. A single vessel supplies the scaphoid, capitate, and the lunate in approximately $20 \%$ of people, which places

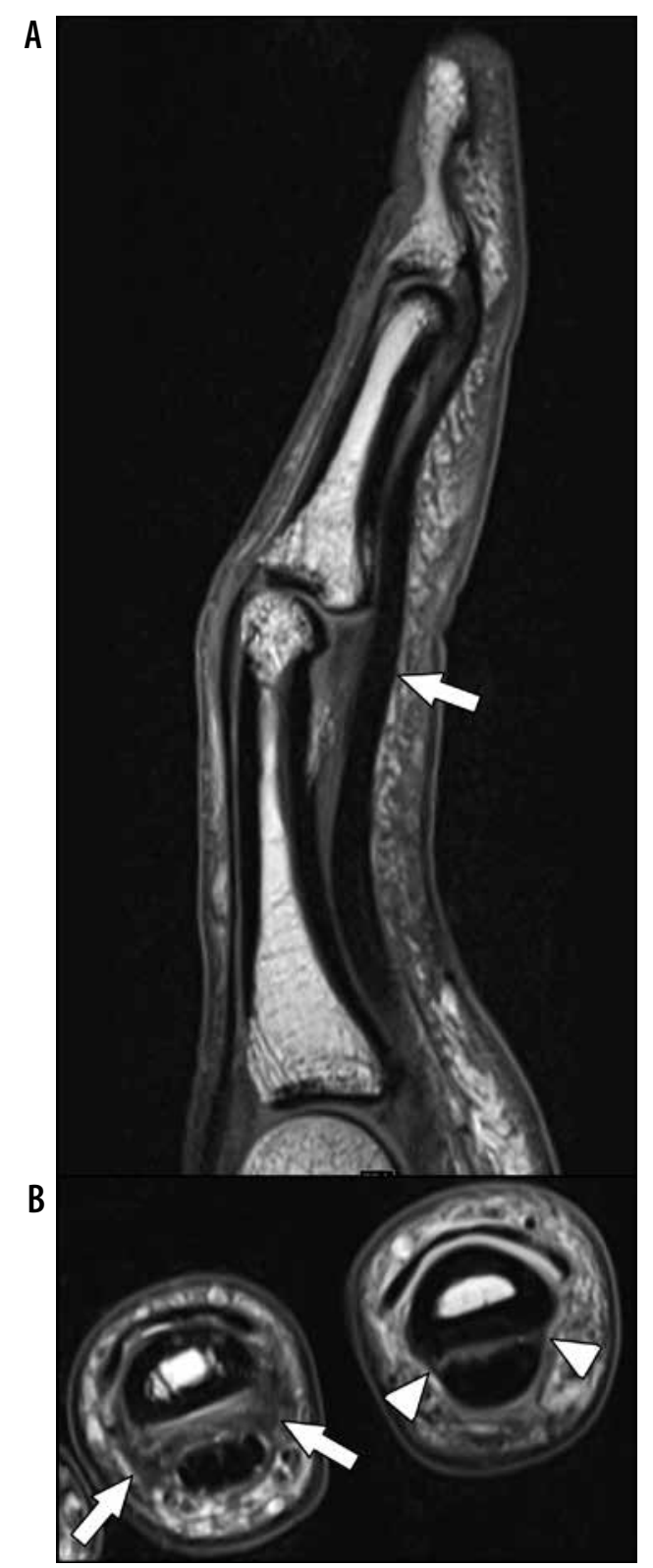

Figure 9. Flexor tendon bowstringing from pulley injury in a 24-year-old male professional baseball player who had injury to the long finger. A) Sagittal T1W non-fat suppressed (NFS) magnetic resonance (MR) image of the finger in mild flexion demonstrates bowstringing of the tendon at the $A 2$ and $A 3$ pulley levels (arrow). The tendon-to-phalanx distance at the A2 pulley measures $3.1 \mathrm{~mm}$, which is abnormal. B) Axial proton density NFS MR image at the level of the middle and ring finger proximal phalangeal shafts shows thickening and intermediate signal within the A2 pulley of the middle finger (arrows), consistent with chronic injury and scarring. The A2 pulley of the ring finger is normal in appearance (arrowheads)

these bones most at risk for developing osteonecrosis [30]. The trapezoid and hamate have two non-articular nutrient arteries but lack consistent intraosseous anastomosis, which also places fracture fragments at risk for osteonecrosis. The trapezium, triquetrum, and pisiform bones in approximately $80 \%$ of people demonstrate redundant blood supply making osteonecrosis in these bones rare [30].

Ulnar variance refers to the relative lengths of the distal radial and ulnar articular surfaces. It can be classified 


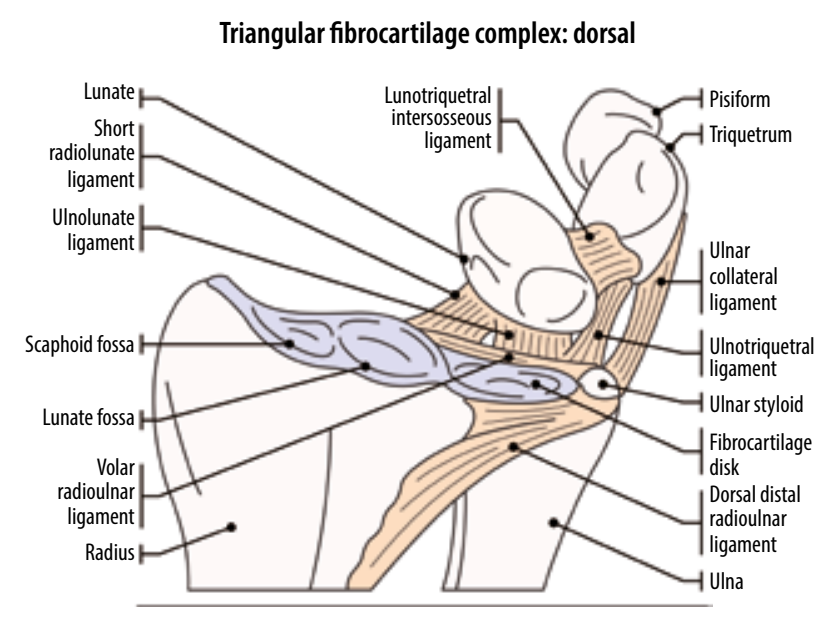

Figure 10. Illustrated diagram of the triangular fibrocartilage complex

as positive (ulnar surface longer), neutral (both articular surfaces of the same length), or negative (ulnar surface shorter). The ulnar styloid process can be variable in length and is independent of ulnar variance. Wrist position can alter the degree of ulnar variance. As a result, although ulnar variance can be suggested on MRI, using this modality to diagnose and measure the degree of variance is inappropriate. Therefore, determination of subtle ulnar variance on MRI should be done with caution [31].

\section{Triangular fibrocartilage complex}

The TFCC lies on the ulnar side of the wrist and acts as the primary stabiliser of the distal radioulnar joint (DRUJ). It is composed of the triangular fibrocartilage disc (TFC), the dorsal and volar radioulnar ligaments, the ulnar collateral ligament (UCL, between the ulnar styloid process and triquetrum), the meniscus homologue, and the extensor carpi ulnaris (ECU) tendon sheath (Figure 10) $[26,32]$. The TFC is thicker peripherally than centrally, giving it a biconcave appearance on sagittal images. It attaches to the distal margin of the sigmoid notch at the ulnar aspect of the distal radius over the articular cartilage. The peripheral attachment of the TFC to the distal ulna is named the triangular ligament and has a proximal and a distal attachment, known as the proximal and distal laminae. The proximal lamina attaches to the distal ulnar fovea at the base of the ulnar styloid. The distal lamina attachment is variable and extends to and beyond the ulnar styloid process; it blends with the fibrous connective tissue of the ECU tendon sheath. The space between the proximal and distal laminae of the triangular ligament is termed the ligamentum subcruentum, which represents loose, vascularised tissue. Increased signal within the ligamentum subcruentum on $\mathrm{PD}$ images can be normal and should not be misinterpreted as a tear [33]. The meniscus homologue (ulnomeniscal homologue) is composed of fibrous tissue between the ulna and triquetrum. It has a common origin with the dorsal radioulnar ligament and inserts on the ulnar aspect of the triquetrum and lunate [26,32].

\section{Intrinsic ligaments}

The wrist consists of many ligaments, classified as intrinsic (interosseous) and extrinsic (capsular). The intrinsic ligaments connect and are interposed between the carpal bones, and are important for maintaining the integrity of the proximal carpal row. The extrinsic ligaments connect the carpal bones either to the radius and ulna or the metacarpal bones. Injuries may result in partial-thickness, fullthickness incomplete, and full-thickness complete tears.

The most important ligaments for supporting the proximal carpal row, and those that are most commonly injured, are the scapholunate (SLL) and lunotriquetral (LTL) ligaments. The SLL is C-shaped and consists of dorsal, central, or membranous, and volar or palmar bands. The dorsal and volar bands are true ligaments; on the other hand, the central component is composed of fibrocartilage. The dorsal band of the SLL is the most important for stability of the wrist [34] and is homogenously low in signal intensity on MRI (Figure 11). The other two bands show intermediate signal resulting from the presence of fibrovascular components. The membranous components of both the SLL and LTL are best depicted on coronal MRA and computed tomography arthrographic images.

The LTL is V-shaped and also made of three components: dorsal, central, or membranous, and volar or palmar bands. The LTL is smaller than the SLL. The volar component blends with the TFCC and is the strongest. However, the dorsal component is the most important in carpal stability.

\section{Extrinsic ligaments}

The extrinsic wrist ligaments are divided into palmar/ volar and dorsal structures (Figure 12). The volar extrinsic wrist ligaments are stronger than the dorsal ligaments and more important for proper wrist function, but show variable anatomy (Figure 12A). The most important ones are the volar radioscaphocapitate ligament, which prevents rotatory subluxation of the scaphoid and the radiolunatotriquetral ligament (also known as the long radiolunate ligament), which connects the distal radius with the proximal carpal row (Figure 12A and C). The radioscaphocapitate ligament originates from the distal aspect of the radial styloid and extends ulnarly and distally to attach to the capitate. The long radiolunate ligament originates from the radial styloid, adjacent to the origin of the radioscaphocapitate ligament, and extends obliquely to attach on the lunate. The deep fibres of this ligament blend with the LTL and attach to the triquetrum [34]. The additional volar extrinsic ligaments are the short radiolunate, radioscapholunate and palmar scaphotriquetral. Two ulnar-sided volar extrinsic wrist ligaments, the volar ulnolunate and volar ulnotriquetral ligaments, originate from the volar radioulnar ligament and insert on the lunate and triquetral bones, respectively (Figure 10). These ligaments contribute to the stability of the TFCC $[26,34]$. 

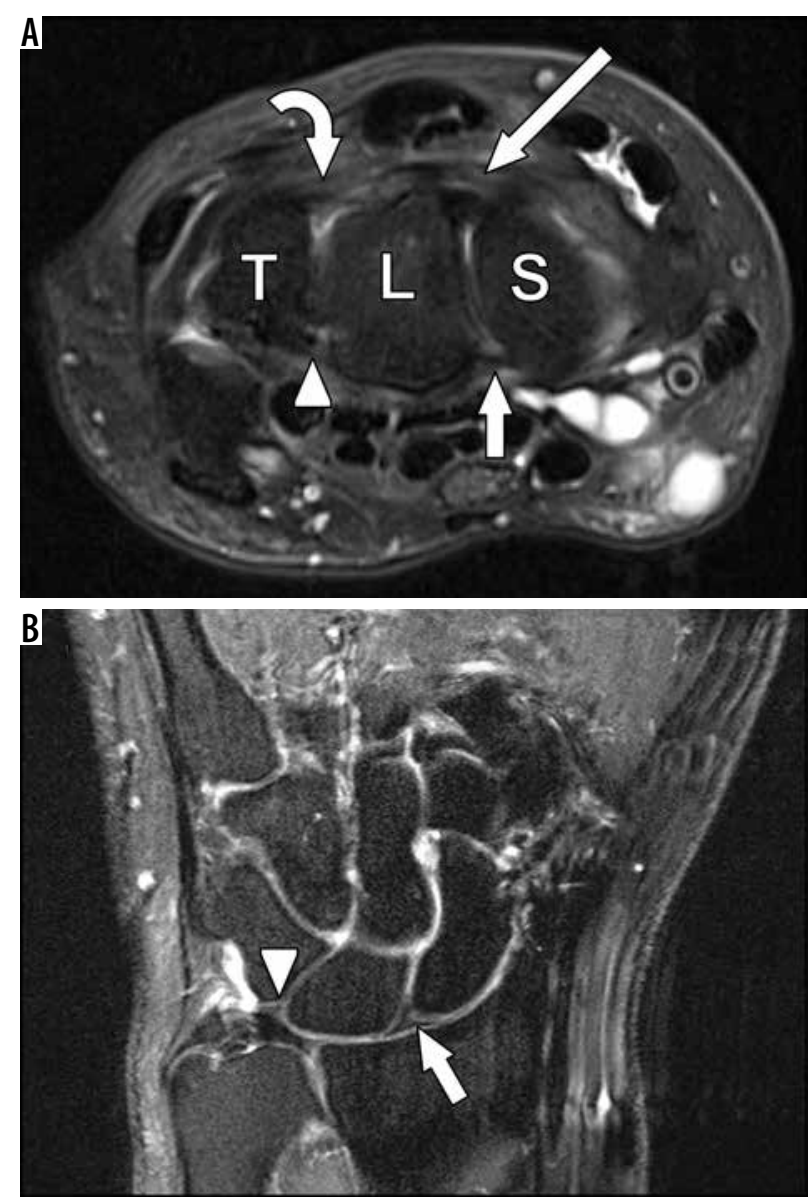

Figure 11. Normal scapholunate (SLL) and lunotriquetral (LTL) ligament anatomy. A) Axial T2W fat-suppressed (FS) magnetic resonance (MR) image through the level of the carpus demonstrate the scaphoid (S), lunate $(\mathrm{L})$, and triquetrum $(\mathrm{T})$. The dorsal bands of the SLL (long arrow) and LTL (curved arrow) are seen. The volar bands of the SLL (short arrow) and LTL (arrowhead) are also noted. B) Coronal T2W FS MR image demonstrates the normal triangular shaped appearance of the central component of the SLL (white arrow) and LTL (arrowhead)

The most important dorsal extrinsic ligament is the dorsal radiocarpal or radiolunatotriquetral ligament. An additional dorsal capsular wrist ligament is the dorsal intercarpal ligament which extends from the scaphoid to the dorsal tubercle of the triquetrum and variably to the second metacarpal base (Figure 12B) [26,34]. Some authors describe an additional dorsal ulnotriquetral ligament.

The radial collateral ligament (RCL) of the wrist is located between the radial styloid and scaphoid, and the UCL is located between the ulnar styloid and triquetrum. These ligaments do not play a significant role in wrist stability (Figure 12B).

Extrinsic wrist ligaments can be depicted with MRI and MRA, including 3D isotropic imaging, and have a striated appearance. All imaging planes are used for their assessment.

\section{Carpal tunnel, including median nerve and flexor tendons}

The carpal tunnel is an anatomic space overlying the volar aspect of the carpal bones (Figure 13A). Its volar surface

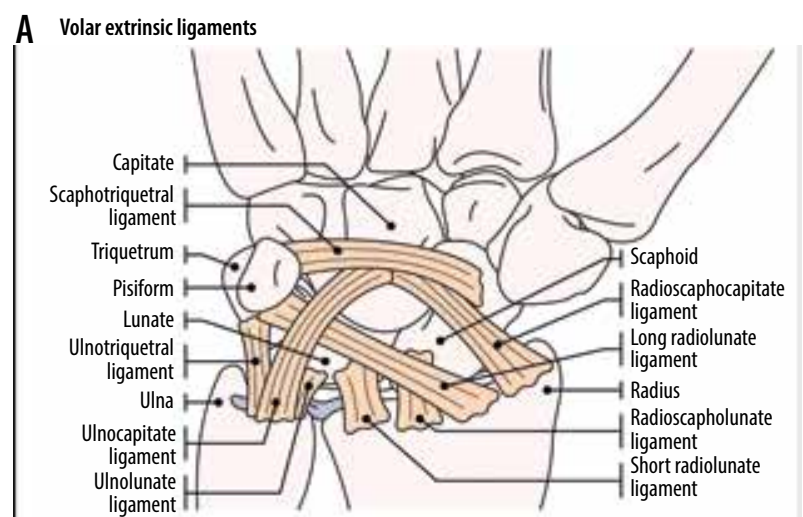

B Dorsal extrinsic ligaments
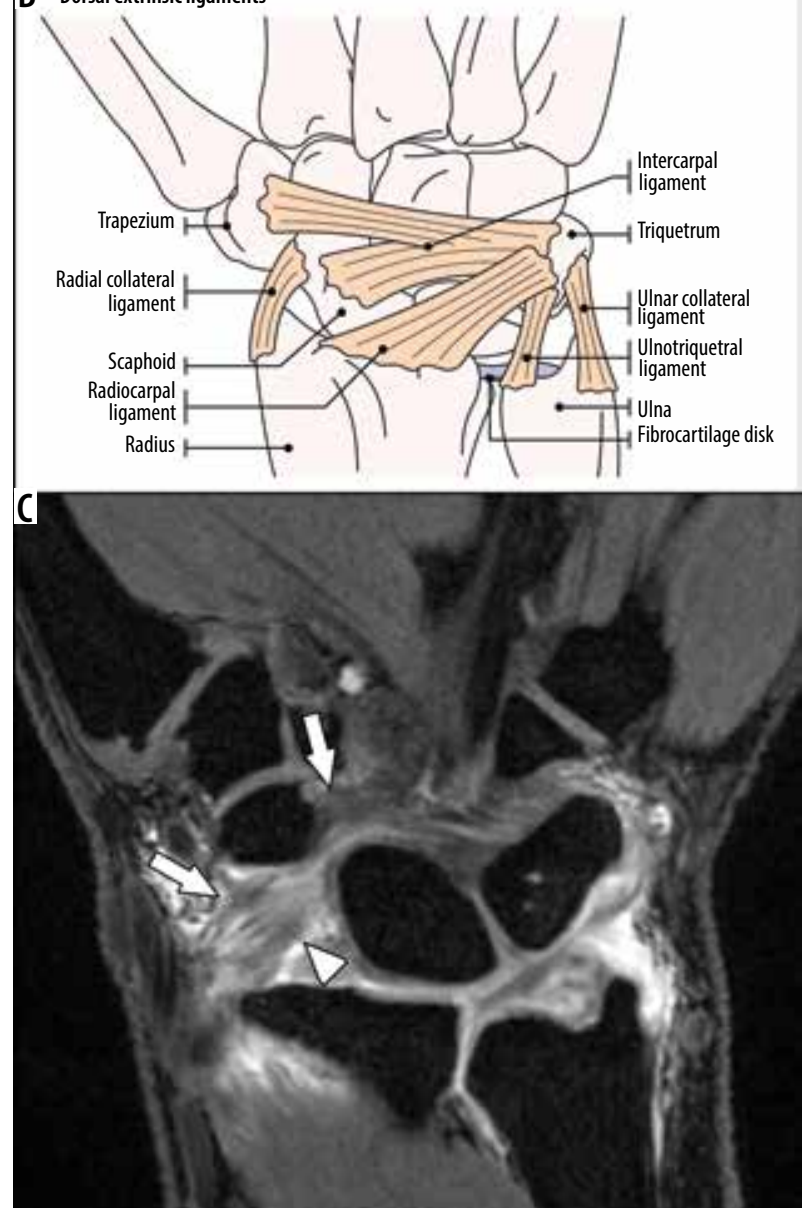

Figure 12. Normal extrinsic wrist ligaments. A) Illustration of the major volar extrinsic ligaments. B) Illustration of the major dorsal wrist extrinsic ligaments and collateral ligaments. C) Coronal 3D T1W fat-suppressed gradient echo post arthrogram magnetic resonance image shows portions of the volar radioscaphocapitate (arrows) and long radiolunate (arrowhead) ligaments

is the flexor retinaculum, which extends from the distal radius to the metacarpal bases. The flexor retinaculum can be divided into three components, including the antebrachial fascia, transverse carpal ligament, and distal aponeurosis between the thenar and hypothenar muscles. The ulnar margin consists of the hook of the hamate, triquetrum, and pisiform bones. The radial margin of the carpal tunnel consists of the scaphoid, trapezium, and the flexor carpi radialis tendon sheath $[26,35]$. 
A Wrist cross-section: cistal

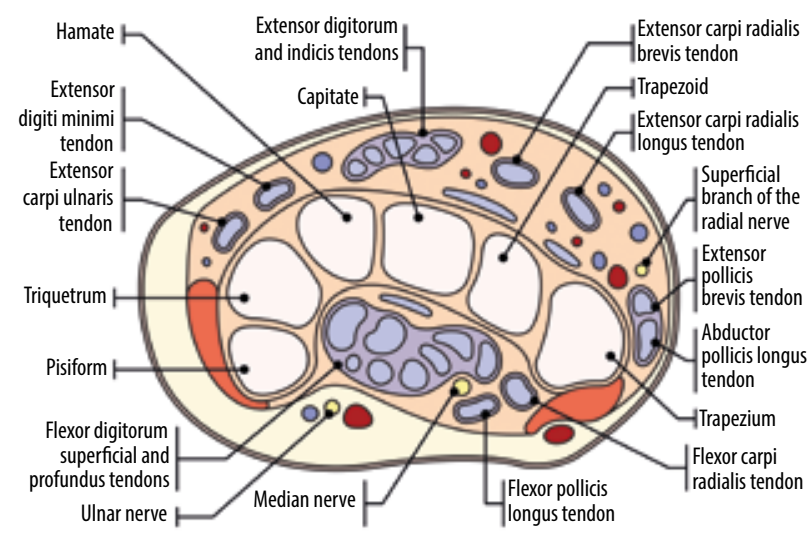

B Wrist cross-section: proximal

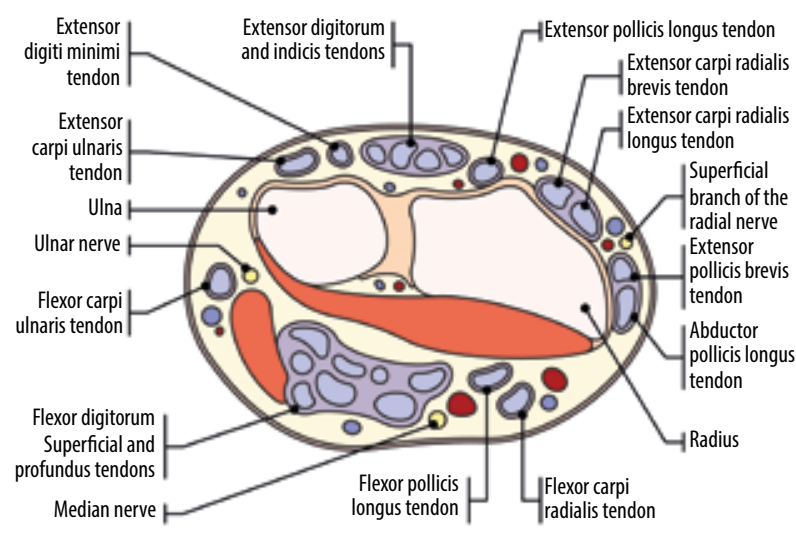

Figure 13. Illustrated diagrams depicting the wrist in cross-section. A) Illustration of the wrist flexor and extensor tendons in the distal wrist at the level of the carpal tunnel. B) Illustration of the wrist flexor and extensor tendons in the wrist at the distal radioulnar level

The carpal tunnel contains nine tendons including the four flexor digitorum superficialis (FDS) tendons, four flexor digitorum profundus (FDP) tendons, and the flexor pollicis longus tendon. The FDS and FDP tendons are located in the ulnar bursa, while the flexor pollicis longus tendon is located within the radial bursa. The ulnar bursa is most frequently in continuity with the fifth digit flexor tendon sheath, while the second to fourth digits usually have separate flexor tendon sheaths. The radial bursa is in continuity with the flexor pollicis longus tendon sheath at the volar aspect of the thumb [36]. There is anatomic variability of the bursae, and the two bursae may communicate with one another or the ulnar bursa may occasionally be continuous with the second to fourth finger flexor tendon sheaths [37]. The flexor carpi radialis tendon has its own osteofibrous tunnel, and is separated from the carpal tunnel by the deep portion of the transverse carpal ligament, which splits along its radial border, surrounding this tendon and bridging across the trapezium.

The palmaris longus tendon is present in $86 \%$ of the population and is located superficially to the flexor retinaculum [38]. It inserts onto the central part of the flexor retinaculum and lower part of the palmar aponeurosis. This muscle assists with wrist flexion; however, it is considered functionally insignificant. Its greatest importance can be to serve as a donor for tendon grafting [39].

The median nerve is the most superficial structure at the radial aspect of the carpal tunnel between the flexor retinaculum and flexor tendons of the index and middle fingers. On MRI, the median nerve has a rounded or slightly flattened appearance with intermediate signal intensity and a relatively constant calibre from DRUJ up to the level of the hook of the hamate.

The flexor tendons are situated at the volar aspect of the wrist and extend distally with the majority attaching onto the metacarpal bones and phalanges. The origins and insertions of the wrist and hand muscles and tendons are described in Table 1.

\section{Extensor tendons}

There are six extensor tendon compartments along the dorsum of the wrist, deep to the extensor retinaculum, which extend distally to attach onto the metacarpal bones and pha-

Table 1. Tendons at the volar aspect of the wrist and han

\begin{tabular}{|c|c|c|}
\hline $\begin{array}{l}\text { Tendons at the volar aspect } \\
\text { of the wrist and hand }\end{array}$ & Origin & Attachment \\
\hline Flexor pollicis longus & Anterior surface of radius and interosseous membrane & Volar aspect of the thumb distal phalangeal base \\
\hline Flexor carpi radialis & Common flexor tendon (ulnar epicondyle of the humerus) & $2^{\text {nd }}$ and $3^{\text {rd }}$ metacarpal bases \\
\hline Flexor digitorum superficialis & $\begin{array}{l}\text { Humeroulnar head: common flexor tendon; radial head: } \\
\text { middle } 1 / 3 \text { of radius }\end{array}$ & $\begin{array}{l}\text { Radial and ulnar sides at the dorsum of the middle } \\
\text { phalangeal shafts of } 2^{\text {nd }}-5^{\text {th }} \text { digits }\end{array}$ \\
\hline Flexor digitorum profundus & $\begin{array}{l}\text { Posterior border of the ulna, proximal two-thirds of medial } \\
\text { border of ulna, interosseous membrane }\end{array}$ & $\begin{array}{l}\text { Volar aspect of the distal phalangeal bases } \\
\text { of the } 2^{\text {nd }}-5^{\text {th }} \text { digits }\end{array}$ \\
\hline Flexor carpi ulnaris & $\begin{array}{l}\text { Common flexor tendon and (ulnar head) from medial border } \\
\text { of olecranon and upper } 2 / 3 \text { of the posterior border of the ulna }\end{array}$ & Pisiform, hook of hamate, and $5^{\text {th }}$ metacarpal base \\
\hline Palmaris longus & Medial epicondyle of the humerus & $\begin{array}{c}\text { Distal half of flexor retinaculum and palmar } \\
\text { aponeurosis }\end{array}$ \\
\hline Pronator quadratus & $\begin{array}{l}\text { Medial side of the anterior surface of the distal one-fourth } \\
\text { of the ulna }\end{array}$ & Anterior surface of the distal one-fourth of the radius \\
\hline
\end{tabular}


Table 2. Tendons at the dorsal aspect of the wrist and hand

\begin{tabular}{|c|c|c|}
\hline Extensor compartment & Origin & Attachment \\
\hline \multicolumn{3}{|l|}{$1^{\text {st }}$} \\
\hline Abductor pollicis longus & $\begin{array}{l}\text { Middle one-third of the posterior surface of the radius, } \\
\text { interosseous membrane, mid-portion of posterolateral ulna }\end{array}$ & Radial side of the base of the first metacarpal \\
\hline Extensor pollicis brevis & $\begin{array}{l}\text { Interosseous membrane and the posterior surface } \\
\text { of the distal radius }\end{array}$ & Base of the proximal phalanx of the thumb \\
\hline \multicolumn{3}{|l|}{$2^{\text {nd }}$} \\
\hline $\begin{array}{l}\text { Extensor carpi } \\
\text { radialis longus }\end{array}$ & $\begin{array}{l}\text { Lower one-third of the lateral supracondylar ridge } \\
\text { of the humerus }\end{array}$ & Dorsum of the second metacarpal base \\
\hline $\begin{array}{l}\text { Extensor carpi } \\
\text { radialis brevis }\end{array}$ & Common extensor tendon (lateral epicondyle of the humerus) & Dorsum of the third metacarpal base \\
\hline \multicolumn{3}{|l|}{$3^{\text {rd }}$} \\
\hline Extensor pollicis longus & $\begin{array}{l}\text { Interosseous membrane and middle part } \\
\text { of the posterolateral surface of the ulna }\end{array}$ & Dorsal aspect of the thumb distal phalangeal base \\
\hline \multicolumn{3}{|l|}{$4^{\text {th }}$} \\
\hline Extensor indicis proprius & $\begin{array}{l}\text { Interosseous membrane and the posterolateral surface } \\
\text { of the distal ulna }\end{array}$ & $\begin{array}{l}\text { Joins the tendon of the extensor digitorum to the second } \\
\text { digit; both tendons insert into the extensor expansion }\end{array}$ \\
\hline Extensor digitorum & Common extensor tendon (lateral epicondyle of the humerus) & $\begin{array}{l}\text { Extensor expansion at the dorsal aspect } \\
\text { of the } 2^{\text {nd }}-5^{\text {th }} \text { digits }\end{array}$ \\
\hline \multicolumn{3}{|l|}{$5^{\text {th }}$} \\
\hline Extensor digiti minimi & $\begin{array}{l}\text { Common extensor tendon } \\
\text { (lateral epicondyle of the humerus) }\end{array}$ & $\begin{array}{l}\text { Joins the extensor digitorum tendon to the } 5^{\text {th }} \text { digit } \\
\text { and inserts into the extensor expansion }\end{array}$ \\
\hline \multicolumn{3}{|l|}{$6^{\text {th }}$} \\
\hline Extensor carpi ulnaris & $\begin{array}{l}\text { Common extensor tendon and the middle one-half } \\
\text { of the posterior border of the ulna }\end{array}$ & Ulnar side of $5^{\text {th }}$ metacarpal base \\
\hline Other & Origin & Attachment \\
\hline Brachioradialis & Upper two-thirds of the lateral supracondylar ridge of the humerus & Radial side of the radial styloid process base \\
\hline
\end{tabular}

langes (Figure 13B). The origins and insertions of the wrist and hand muscles and tendons are described in Table 2.

\section{Radial and ulnar nerves}

The radial nerve provides motor and sensory function to the forearm, wrist, and hand via the deep and superficial branches. The superficial radial nerve provides sensory function of the dorsolateral aspect of the hand (Figure 13). In the distal forearm, the superficial radial nerve courses posterior to the radial styloid and first extensor compartment. Given the superficial nature of the nerve in this location, it may be prone to traumatic injuries from distal radius fractures [40].

The ulnar nerve at the level of the wrist lies within a fibro-osseous tunnel known as Guyon's Canal (Figure 13A). The canal is formed by the flexor retinaculum and palmar carpal ligament within the proximal aspect of the hypothenar eminence. The canal it begins at the proximal margin of the transverse carpal ligament at the level of the proximal pisiform and ends at the aponeurotic arch of the hypothenar musculature at the level of the hook of the hamate. The ulnar artery and occasionally communicating veins travel with the nerve through the canal, which otherwise contains fat only [26,39].

\section{Common anatomic variants}

There are numerous normal osseous, muscular, and nerve anatomic variants that can either mimic pathology or be a cause of symptoms. Listing each described variation is beyond the scope of this article. However, the most common and clinically relevant variants are described here.

Within the bones there are variant morphologies of some of the carpal bones, including developmental coalitions and numerous reported developmental ossicles. For instance, a lunotriquetral coalition is the most common carpal coalition, with a prevalence of $0.1 \%$, and it represents a congenital fusion of the lunate and triquetral bones [41]. Osseous coalitions of the lunate and triquetrum are typically asymptomatic. However, fibrocartilaginous LTL coalitions can be associated with atypical ulnar-sided wrist pain.

Two lunate morphologies have been described. A type I lunate has a single distal facet with the capitate and does not articulate with the hamate, whereas a type II lunate has 
two distal facets with the radial facet articulating with the capitate and ulnar facet articulating with the hamate. This can be associated with focal lunohamate osteoarthritis and ulnar-sided wrist pain.

There are numerous accessory ossicles of the wrist that can be mistaken for pathology or occasionally be a source of symptoms. Two of the most commonly encountered ossicles are the os styloideum and the os hamuli. The os styloideum is a dorsal accessory ossicle between the second and third metacarpal bases that can be fused with the metacarpal base; it has been associated with carpal bossing and dorsal hand pain. The os hamuli forms when the hook of the hamate fails to fuse with the body of the hamate. The primary differential consideration is a chronic hook of hamate fracture; however, if the bone has smooth, well-corticated, and rounded margins it more likely represents an accessory ossicle. Other, ossicles, including the lunula, os triangulare, os trapezium secundarium, and os epilunate, are also occasionally observed but are less commonly clinically relevant.

In some individuals, additional accessory tendons and muscles may be present and should not be mistaken for pathology. For instance, the accessory abductor digiti minimi is the most common accessory muscle of the wrist with a prevalence of up to $24 \%$. The muscle is radial and volar to the pisiform and inserts with the abductor digit minimi on the ulnar margin of the fifth metacarpal base. It can be readily identifiable as muscle tissue projecting into Guyon's canal at the level of the pisiform because there normally should not be any muscle in Guyon's canal at that level [42].

The extensor digitorum brevis manus is an accessory muscle that occurs in the fourth extensor compartment and can be unilateral or bilateral. The origin of the muscle can be variable, although it most commonly arises from the dorsal wrist capsule deep to the extensor retinaculum [42]. It usually inserts onto the extensor hood of the index or middle fingers but can occasionally insert onto the fourth and fifth fingers $[43,44]$. Clinically, it can be mistaken as a mass along the dorsal aspect of the wrist.

A bifid median nerve is a common anatomic variant with an incidence of approximately $2.8 \%$. The median nerve can bifurcate into two nerve bundles in the distal forearm and appear as a bifid median nerve in the carpal tunnel. The bifid median nerve can also appear with an accessory artery, the persistent median artery, which lies between the two nerve bundles. Identifying this variation as well as the presence of the accessory artery can be of importance in avoiding injury to these structures during carpal tunnel release [45].

\section{Internal derangement of the wrist}

\section{Bones}

\section{Occult fractures and their complications}

MRI is the best imaging modality for evaluating radiographically occult wrist fractures, fracture complications, and osseous contusions (Figure 1). The scaphoid is important for carpal stability and acts as a stabiliser between the two carpal rows. Anatomic snuff box tenderness is a sensitive test for scaphoid fractures, but pain and tenderness over the scaphoid tubercle are more specific. Clinical examination alone is unable to assess for complications of scaphoid fractures.

Scaphoid fractures account for approximately $60-90 \%$ of all wrist fractures, and $12 \%$ of scaphoid fractures fail to heal and require surgical treatment $[26,46]$. Due to the unique blood supply of the scaphoid, fractures through the proximal pole are predisposed to delayed union, nonunion, and osteonecrosis. Radiography, including posteroanterior (PA), lateral, semipronated oblique and PA with ulnar deviation views, is the first-line imaging modality for detecting complications related to scaphoid fractures. However, MRI is more sensitive in detecting radiographically occult fracture lines and bone marrow oedema. If MRI demonstrates a fracture line with cyst formation on either side, there is suggestion of nonunion.

Scaphoid nonunion advanced collapse (SNAC) wrist occurs when persistent nonunion of the scaphoid results in the development of osteoarthritis and functional impairment about 5-10 years after injury. "Humpback deformity" is secondary to malunion of a scaphoid waist fracture with volar angulation of the proximal and distal poles. The angulated dorsal surface of the scaphoid forms the palpable "humpback" [47].

Treatment of scaphoid fracture complications varies depending on the ability to tolerate surgery and the viability of the bone. Patients who are unable to tolerate surgery for scaphoid nonunion may be candidates for non-operative management with casting for 4-6 weeks. In scaphoid nonunion with preserved vascularity, a non-vascularised bone graft fixated with screws is able to achieve successful union. If there is poor blood supply or osteonecrosis seen on MRI and/or absence of bleeding at surgery, vascularised bone grafting is performed. Scaphoid nonunion with osteoarthritis requires salvage procedures such as limited or total wrist arthrodesis, proximal row carpectomy, scaphoid excision, or arthroplasty.

MRI is also useful in detecting other, less frequent fractures involving the carpal bones. Hook of hamate fractures can occur by direct trauma or avulsive force from the transverse carpal ligament. These avulsion injuries are associated with racquet sports and golf [47]. Untreated fractures can progress to nonunion and may rarely require surgical excision for symptomatic relief [47].

\section{Osteonecrosis}

\section{Scaphoid}

Preiser's disease is the idiopathic form of osteonecrosis of the scaphoid bone, although most cases have a history of repetitive micro-injuries or overloading [11]. However, most cases of scaphoid osteonecrosis occur fol- 
lowing proximal pole or waist fractures, and osteonecrosis has been reported in approximately $30 \%$ of scaphoid waist fractures and $80-100 \%$ when the fracture is located in the proximal pole [46]. Clinical examination cannot alone detect complications of scaphoid fractures. MRI is useful for determining the presence of osteonecrosis. If MRI demonstrates preserved normal fatty signal in the proximal fracture fragment, the finding strongly suggests viable bone marrow. However, mummified fat in early osteonecrosis may demonstrate the "normal" high signal intensity of fat on T1W images without fat saturation resulting in false negative findings. Otherwise, early osteonecrosis demonstrates low signal intensity of necrotic bone with respect to surrounding normal bones on T1W images and variable signal on fluid-sensitive, FS images. Contrast enhancement of the bone marrow suggests preserved vascularity. Homogeneous enhancement suggests viable bone marrow, while the absence of enhancement suggests osteonecrosis, and heterogeneous enhancement may represent the coexistence of osteonecrosis with variable viable tissue (Figure 5). However, fibrous tissue ingrowth can result in some enhancement and lead to false negative interpretation [48-50].

\section{Lunate}

Kienböck disease is osteonecrosis of either a part or all of the lunate. Osteonecrosis may result from repetitive microtrauma, interruption of blood supply including acute fracture, hypercoagulability, steroid use, altered biomechanics in association with negative ulnar variance, decreased radial inclination, and type I lunate morphology. A type II lunate with its extra articulation to the hamate may resist against fractures. Kienböck disease typically presents with dorsal-sided pain, restricted motion, weakness, and swelling. On clinical examination, there is focal tenderness between the extensor digitorum and extensor carpi radialis tendons with provoked pain on extension. Isolated lunate fractures are very rare, accounting for ap-

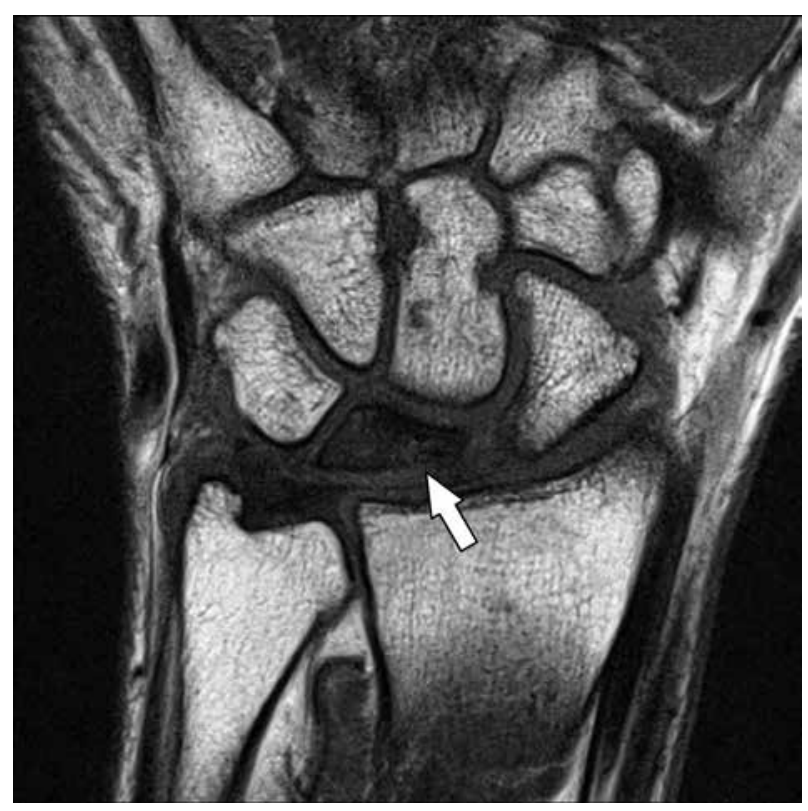

Figure 14. Kienböck Disease. 44-year-old female with a few months of left wrist pain. Coronal T1W magnetic resonance image shows diffuse low T1 signal throughout the lunate (arrow), most notable at its radial aspect, compatible with osteonecrosis with partial collapse. Ulnar negative variance is also suggested on this image

proximately $1-2 \%$ of wrist fractures [51]. The proximal pole of the lunate can be predisposed to ischaemia because it lacks soft tissue attachments. Palmar pole fractures can result in osteonecrosis due to interruption of the single arterial supply, which is observed in up to $20 \%$ of the population [26,31]. MRI findings of lunate osteonecrosis are those described previously when discussing scaphoid osteonecrosis (Figure 14). The Lichtman staging of Kienböck disease is important for patient management (Table 3).

\section{Ulnolunate abutment and ulnar impingement}

Ulnolunate abutment, also known as ulnocarpal loading or ulnar impaction syndrome, is characterised by ulnar wrist

Table 3. Lichtman staging of kienböck disease

\begin{tabular}{|c|c|c|c|}
\hline Stage & Radiographic/CT findings & MRI findings & Management \\
\hline I & Normal morphology & Normal morphology, BME & Conservative/Immobilization \\
\hline$\|$ & $\begin{array}{l}\text { Normal morphology, } \\
\text { bone marrow sclerosis }\end{array}$ & $\begin{array}{l}\text { Normal morphology, low signal on T1W and variable } \\
\text { on fluid-sensitive sequences }\end{array}$ & $\begin{array}{l}\text { Ulnar lengthening or radial } \\
\text { shortening } \pm \text { core decompression }\end{array}$ \\
\hline Illa & $\begin{array}{l}\text { Collapse of lunate bone, } \\
\text { radioscaphoid angle }<60^{\circ}\end{array}$ & $\begin{array}{c}\text { Collapse of lunate bone, low signal on T1W and variable } \\
\text { on fluid-sensitive sequences }\end{array}$ & $\begin{array}{l}\text { Ulnar lengthening or radial } \\
\text { shortening } \pm \text { core decompression }\end{array}$ \\
\hline Illb & $\begin{array}{l}\text { Collapse of lunate bone, } \\
\text { radioscaphoid angle }>60^{\circ}\end{array}$ & $\begin{array}{c}\text { Collapse of lunate bone, low signal on T1W and variable } \\
\text { on fluid-sensitive sequences }\end{array}$ & $\begin{array}{l}\text { Proximal row carpectomy, } \\
\text { arthrodesis } \pm \text { lunate excision }\end{array}$ \\
\hline Illc & $\begin{array}{l}\text { Collapse of lunate bone, } \\
\text { coronal lunate fracture (chronic) }\end{array}$ & $\begin{array}{l}\text { Collapse of lunate bone, low signal on T1W and variable } \\
\text { on fluid-sensitive sequences, Coronal lunate fracture (chronic) }\end{array}$ & $\begin{array}{l}\text { Proximal row carpectomy, } \\
\text { arthrodesis } \pm \text { lunate excision }\end{array}$ \\
\hline IV & $\begin{array}{l}\text { Radiocarpal and midcarpal } \\
\text { osteoarthritis }\end{array}$ & $\begin{array}{l}\text { Radiocarpal and midcarpal osteoarthritis, low signal on T1W } \\
\text { and variable on fluid-sensitive sequences }\end{array}$ & $\begin{array}{l}\text { Proximal row carpectomy, } \\
\text { arthrodesis } \pm \text { lunate excision }\end{array}$ \\
\hline
\end{tabular}

CT - computed tomography, MRI - magnetic resonance imaging, BME - ???????????? ????????????? 


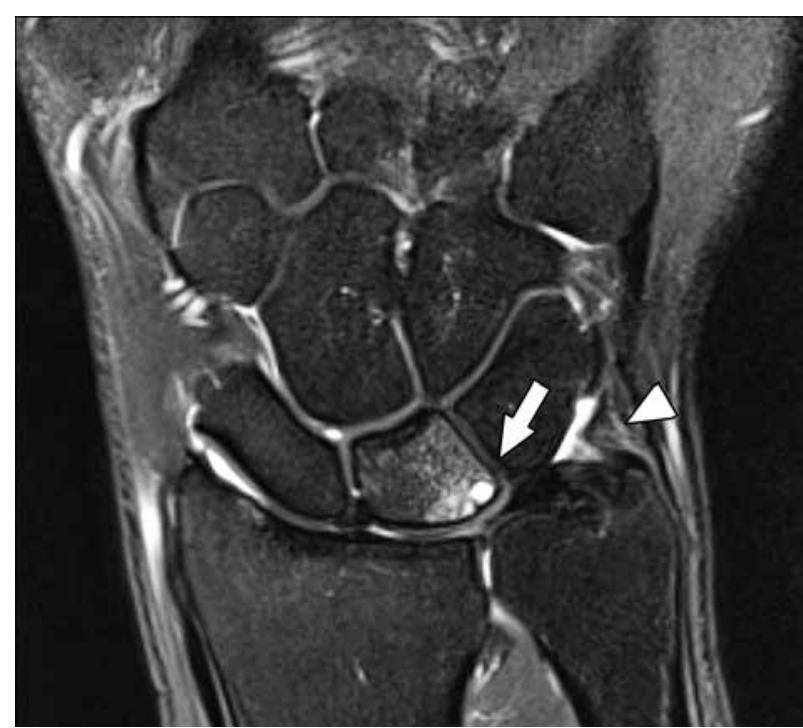

Figure 15. Ulnolunate abutment. 47-year-old male with bilateral wrist pain, left greater than right. Coronal T2W fat-suppressed magnetic resonance image shows bone marrow oedema in the lunate with subchondral cyst-like changes along the proximal ulnar aspect (arrow). There is significant central triangular fibrocartilage disc (TFC) thinning although no perforation was seen. These findings can be seen with ulnolunate abutment. The peripheral TFC appears normal. Note the intact meniscus homologue (arrowhead)

pain, swelling, and limited range of motion. The chronic impaction between the ulnar head and TFCC, lunate, and triquetrum can result in a spectrum of pathologic changes including degenerative tearing of the TFCC, lunate/triquetral chondromalacia, tearing of the LTL, or osteoarthritis of the ulnocarpal and DRUJ (Figure 15). These findings most commonly occur with ulnar positive variance but can occasionally occur with neutral or negative variance [52].

Ulnar impingement is a distinct entity from ulnolunate abutment. It is a syndrome characterised by a shortened distal ulna (negative ulnar variance) that impinges on the distal radius proximal to the sigmoid notch. The clinical findings are similar to those of ulnolunate abutment; however, there is pain on pronation and supination of the forearm and with compression of DRUJ. MRI findings include erosive changes at the level of the radius with osseous remodelling. MRI can be useful to detect earlier findings such as bone marrow oedema prior to the remodelling [52].

Table 4. Palmer's classification of TFCC injuries

\begin{tabular}{|l|l|l|l|}
\hline \multicolumn{2}{|c|}{ Class 1. Traumatic injuries } & \multicolumn{2}{|c|}{ Class 2. Degenerative injuries } \\
\hline 1A & Central perforation or tear & 2A & TFCC wear and thinning \\
\hline 1B & Ulnar avulsion & 2B & $\begin{array}{l}\text { Lunate and/or ulnar } \\
\text { chondromalacia + 2A }\end{array}$ \\
\hline 1C & $\begin{array}{l}\text { Distal avulsion (origin } \\
\text { of ulnolunate and } \\
\text { ulnotriquetral ligaments) }\end{array}$ & 2C & TFCC perforation + 2B \\
\hline 1D & Radial avulsion & 2D & Ligament disruption + 2C \\
\cline { 2 - 4 } & 2E & Ulnocarpal and DRUJ arthritis + 2D \\
\hline
\end{tabular}

TFCC - triangular fibrocartilage complex, DRU - distal radioulnar joint

\section{Triangular fibrocartilage complex}

TFCC injuries most commonly occur from axial loading upon the extended wrist in pronation. Racquet sport athletes may present with TFCC injuries due to repetitive distraction loading on the volar aspect of the wrist, and ulnar positive variance can predispose to TFCC injuries. Patients with TFCC pathology present with ulnarsided wrist pain, variable instability, and often a clicking or snapping sensation with rotation [26,32]. Due to the complex anatomy of the TFCC, high-resolution MRI with high SNR and high contrast resolution is required for accurate assessment. No specific imaging features are able to discriminate traumatic from degenerative tears.

TFCC injuries can be organised by Palmer's Classification (Table 4) into either traumatic/acute (Class 1) or degenerative/chronic (Class 2). Class 2 tears are often the result of ulnolunate abutment from positive ulnar variance, rarely an elongated ulnar styloid process, or secondary to ulnar lengthening from radial fracture malunion. Class IA lesions appear as 1-2 mm sagittally-oriented tears ulnar to the radial aspect of TFC (Figure 16). These tears are in the avascular portion of TFC and are usually debrided due to poor healing [53]. Class IB lesions are traumatic avulsions from its attachment at the ulnar fovea proximal or distal laminae (Figure 17). These tears can be associated with fractures through the base of the ulnar styloid and injury to the palmar and dorsal radioulnar ligaments. The location of the tear is in the well-vascularised periphery of TFC and may heal. This is the most commonly missed tear. Excessive fluid accumulation and focal synovitis can be indirect signs of a tear. However, the intermediate to increased $\mathrm{T} 2 \mathrm{~W}$ signal of the ligamentum subcruentum can mimic a tear in this region [26,53]. Class IC lesions are traumatic avulsions from the peripheral volar attachments at the lunate or triquetral bones, specifically the ulnolunate and ulnotriquetral ligaments. If abnormal signal is seen within the ligaments on fluid-sensitive sequences, a tear is likely. The most advanced of these are Class ID lesions, which are traumatic avulsions from the radial attachment in the region of the sigmoid notch. They may be associated with distal radius fractures. These lesions are also usually debrided because the tissue is relatively avascular, which results in poor healing $[26,53]$.

Class IIA lesions represent degenerative wear and thinning of the articular disk without perforation. Focal abnormal signal intensity not extending to the surface of TFC probably represents mucoid degeneration. Class IIB lesions demonstrate progressive degeneration with findings of IIA lesions and additional chondromalacia of the lunate, triquetrum, or distal ulna without perforation. Class IIC lesions demonstrate even more progressive change than IIB lesions with a frank perforation of TFC, and Class IID lesions demonstrate LTL perforation as well as all of the findings of class IIC lesions. These lesions result in the LTL instability. Finally, Class IIE lesions are 


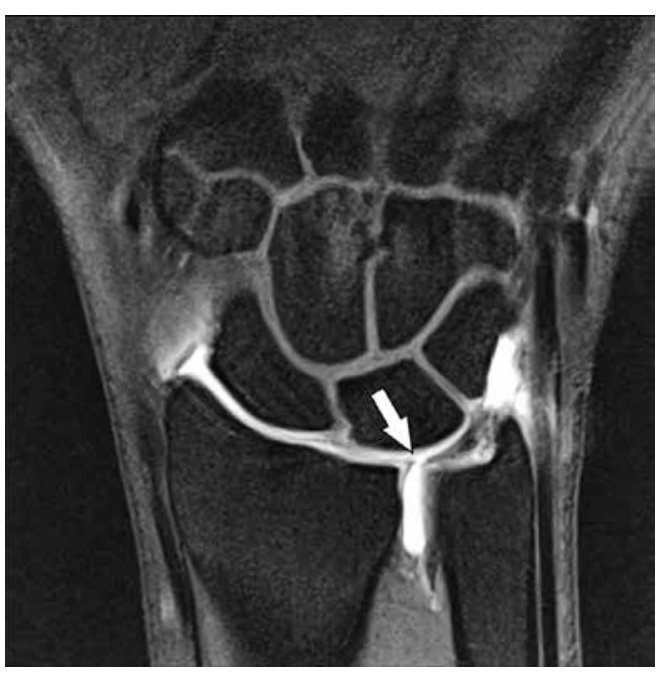

Figure 16. Central triangular fibrocartilage disc (TFC) tear. 26-year-old male with ulnar-sided right wrist pain after injury 9 months prior. Coronal T1W fat-suppressed magnetic resonance arthrography image after single compartment radiocarpal joint injection shows a full-thickness perforation through the central TFC disc (arrow). T1W hyperintense contrast is seen extending through the defect and into distal radioulnar joint, consistent with a Palmer $1 \mathrm{~A}$ lesion

Class IID lesions with ulnocarpal and occasional distal radioulnar osteoarthritis. If the LTL is sufficient, they can be treated with debridement and pinning. However, if this ligament is completely absent, arthrodesis may be necessary [53].

Communicating tears are full thickness defects in which single injection MRA shows extension of contrast from the radiocarpal to the DRUJ (Figure 6 and 16). Non-communicating tears comprise partial-thickness defects. Degenerative tears and perforations are age-related and usually occur in the central disc. Radial/central communicating tears are often bilateral and can be asymptomatic [26,32,33].

Direct MRA has the highest accuracy in detecting TFC tears, especially for central and radial-sided (Palmer IA, ID, and II) tears. Moderate accuracy is present for peripheral tears at the ulnar attachment (Palmer IB, IC); however, MRA is still considered superior to PD/T2W FS images due to the presence of fibrovascular tissue [26].

\section{Intrinsic ligaments}

The SLL and the LTL are the most important ligaments that support the proximal carpal row. Furthermore, they are the most commonly injured ligaments, and their disruption can lead to altered biomechanics. The most frequent mechanism of injury is extension, ulnar deviation, and carpal supination. Repetitive stress to the SLL from crutch walking and ulnar impingement of the LTL can also result in injury $[26,33,34]$.

Tears of the dorsal SLL band at its lunate attachment are usually symptomatic and traumatic rather than degenerative. Degenerative perforations of the central SLL and LTL increase with age and can be asymptomatic. Tears

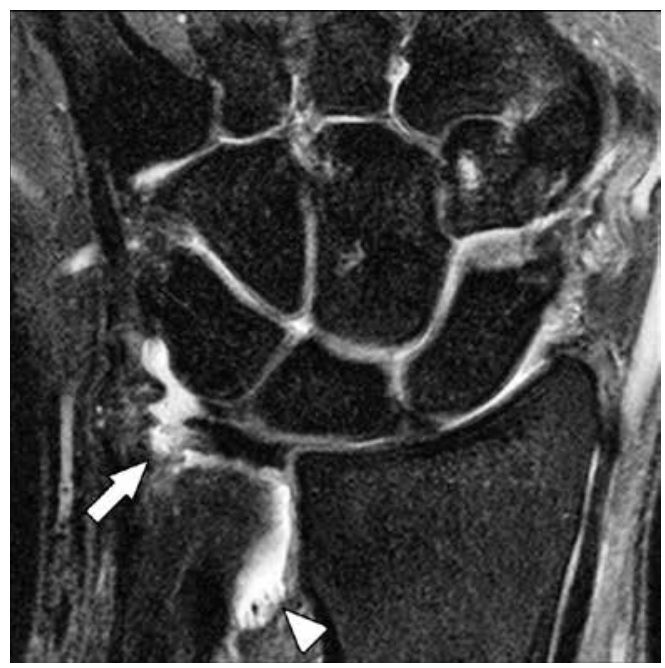

Figure 17. Peripheral triangular fibrocartilage complex (TFCC) tear. 49-yearold male with dorsal and ulnar-sided wrist pain following trauma. Coronal T2W fat-suppressed magnetic resonance image shows partial tearing of the triangular ligament of the peripheral TFCC at its ulnar styloid attachment (arrow) and a small distal radioulnar effusion with synovial proliferation, which commonly accompanies TFCC tears (arrowhead), consistent with a Palmer $1 \mathrm{~B}$ lesion. Additional injuries to the distal radioulnar ligament and extensor carpi ulnaris tendon subsheath are not shown

of the SLL result in rotatory subluxation of the scaphoid and dorsal intercalated segmental instability (DISI) with dorsal tilting of the lunate (Figure 18). An SLL angle $>80^{\circ}$ is diagnostic for DISI. This is best measured on true lateral wrist radiographs. Although it can be estimated on MRI, MRI measurements may be unreliable and highly dependent on patient positioning. Zanetti et al. found the mean measurement of the SLL angle on sagittal MRI to be closely correlated with measurements taken on lateral radiographs when the wrist was positioned in $15^{\circ}$ of radial deviation. However, when the wrist was placed in the neutral position the measurement could differ from the radiographic measurement by $14^{\circ}$, and when the wrist was placed in $15^{\circ}$ of ulnar deviation, the measurement could differ by $32^{\circ}$ [54]. DISI can result in abnormal loading on the wrist and eventually result in scapholunate advanced collapse (SLAC) wrist, which is characterised by proximal migration of the capitate and osteoarthritis of the radiocarpal and intercarpal joints. The abnormal SLL may demonstrate fraying, irregular margins, increased signal on fluid-sensitive sequences, or fibre discontinuity. These findings may be present with or without widening of the SLL interval. Full-thickness tears will allow abnormal communication of contrast between the radiocarpal and midcarpal joints $[26,33,34]$.

Disruption of the LTL results in volar intercalated segmental instability (VISI) with volar tilting of the lunate. This finding can be a normal variant in patients with joint laxity. The SLL angle $<30^{\circ}$ and capitolunate angle $>30^{\circ}$ are suggestive of VISI. Similar to the SLL, the dorsal band is best seen on axial imaging and the membranous component is best seen on coronal imaging $[26,33,34]$. The lunotriquetral articulation is usually obliquely ori- 


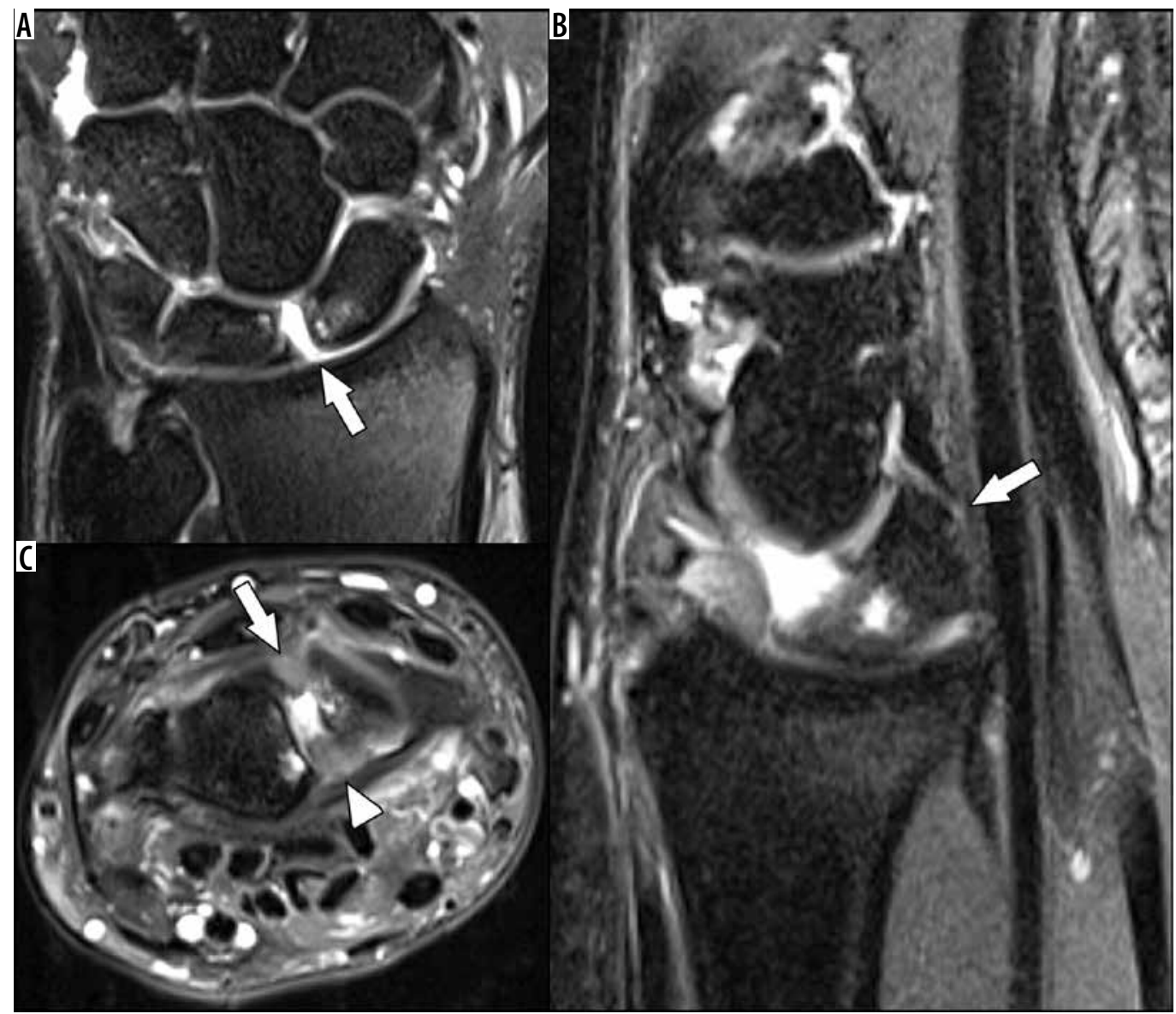

Figure 18. Scapholunate ligament (SLL) tear with dorsal intercalated segmental instability (DISI). 42-year-old male with left wrist pain after a fall. A) Coronal T2W fat-suppressed (FS) magnetic resonance (MR) image shows full-thickness tearing of the central band of the SLL (arrow) with widening of the scapholunate interval. B) Sagittal T2W FS MR image shows dorsal tilt of the lunate (arrow) compatible with DISI. C) Axial T2W FS MR image shows that the dorsal and volar bands of the SLL (arrow and arrowhead, respectively) are poorly defined and thickened, compatible with high-grade tearing

ented with respect to the short axis of the wrist. If there is clinical concern for LTL injury, an oblique axial sequence that is perpendicular to the LTL articulation may be helpful to improve characterisation of the dorsal and volar bands of the ligament.

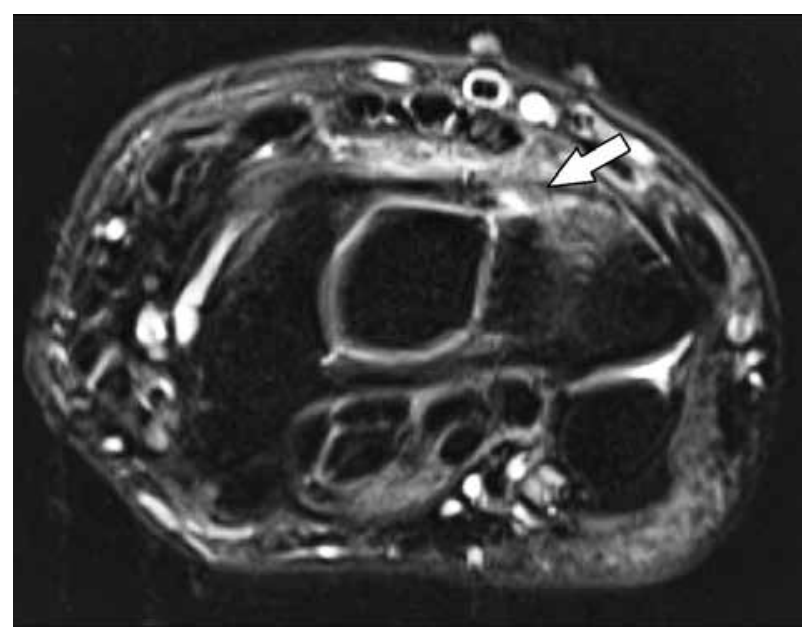

Figure 19. Dorsal intercarpal extrinsic ligament sprain. 47-year-old female with right-side dorsal wrist pain after fall. Axial T2W fat-suppressed magnetic resonance image shows an oedematous and ill-defined dorsal intercarpal ligament especially along its triquetral attachment (arrow)

\section{Extrinsic ligaments}

Tears of the volar extrinsic ligaments may contribute to DISI. The ulnar-sided volar ligaments contribute to the stability of the TFCC. Peripheral tears of the TFCC can result in injury to the volar ulnolunate and ulnotriquetral ligaments. Tears of the dorsal radiocarpal ligaments can lead to VISI, while a tear of the dorsal intercarpal ligament can lead to DISI.

Injuries of the extrinsic ligaments can be concurrently found with intrinsic ligament injury and carpal instability. Torn ligaments demonstrate discontinuity of fibres, while partial tears would show irregularity of the fibres and/or increased signal on fluid-sensitive sequences (Figure 19) [55].

\section{Flexor tendon injuries}

Flexor tendon injuries in the hand can be classified into five anatomic zones (Figure 20). The wrist is composed of zone IV, the tendons within the carpal tunnel, and Zone V, the tendons extending from the myotendinous junction to the proximal aspect of the carpal tunnel. Zone IV injures are rare due to the protective nature of the transverse carpal ligament. However, a crush injury could 
result in damage to the carpal tunnel flexor tendons and the median nerve. Zone $\mathrm{V}$ injuries can result in damage to the ulnar and radial arteries, ulnar and median nerves, as well as multiple flexor tendons [56]. Flexor tendon tenosynovitis can occasionally be seen in the setting of inflammatory arthritis or in athletes with overuse injuries, such as those who play racquet sports or golf [26]. The section on the lesser fingers further discusses the various flexor tendon zones and related injuries in greater detail.

\section{Extensor tendon injuries}

The most common form of tenosynovitis is known as DeQuervain's tenosynovitis and involves the first extensor compartment tendons, including the abductor pollicis longus and extensor pollicis brevis tendons (Figure 21). DeQuervain's tenosynovitis typically presents in middleaged women bilaterally, young mothers carrying babies, and racquet sport athletes. Patients present with pain and swelling over the radial styloid process with increased pain during thumb extension and abduction $[26,57]$.

Proximal intersection syndrome is a usually chronic, overuse, inflammatory condition affecting the dorsal radial aspect of the distal forearm $4-5 \mathrm{~cm}$ proximal to the radiocarpal joint, where the first and second extensor compartment tendons cross each other. This syndrome results from repetitive friction that can lead to tenosynovitis, stenosing tenosynovitis, and occasionally adventitial bursitis. The condition is associated with rowing and racquet sports and is less commonly from direct trauma $[26,57]$.

The ECU tendon can present with tenosynovitis, tendinopathy, subluxation, or dislocation due to tendon sheath disruption and tendon rupture (Figure 22). Although it can be seen in normal individuals, ECU tendon subluxation can also be related to an acute injury, and the patient history and physical examination may be needed to diagnose pathologic subluxation [26,57].

Rheumatoid arthritis (RA) frequently presents with tenosynovitis and tendinopathy because the wrist tendons are surrounded by a synovial sheath or bursa. However, they rarely present with tendon tears. Approximately 50$64 \%$ of RA patients demonstrate extensor tendon involvement, with the ECU being the most commonly affected tendon in patients with early RA. On MRI, tendinopathy is characterised by heterogeneous high signal intensity and thickening on fluid-sensitive sequences, and active tenosynovitis will demonstrate tendon sheath effusions with enhancement [57].

Distal intersection syndrome, also known as decussation syndrome, is caused by the third extensor compartment tendon crossing the second compartment tendons just distal to Lister's tubercle, which arises from the dorsum of the distal radius (Figure 23). The second and third extensor compartment tendon sheaths communicate via a foramen, which can allow the spread of inflammation between the two. Patients present with pain and swell-

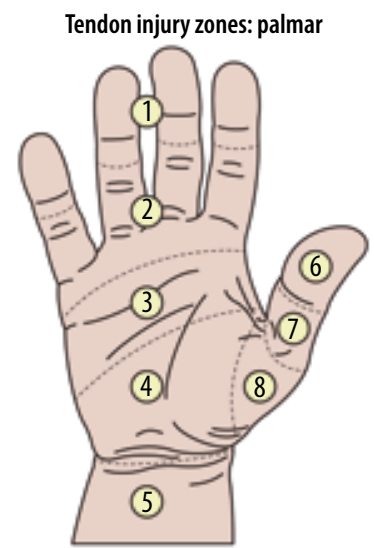

1 - Distal to FDS insertion on midpoint of middle phalanx

$2-$ FDS insertion to level of A1 pulley

3 - Proximal aspect of A1 pulley to origin of lumbricals from FDP tendons

4 - Carpal tunnel

5 - Proximal aspect of transverse carpal ligament to musculotendinous junction

6 - Distal to thumb IP joint

7 - Thumb A1 pulley to IP joint

8 - Thenar eminence

Figure 20. Illustrated schematic of the flexor and extensor tendon zone injuries

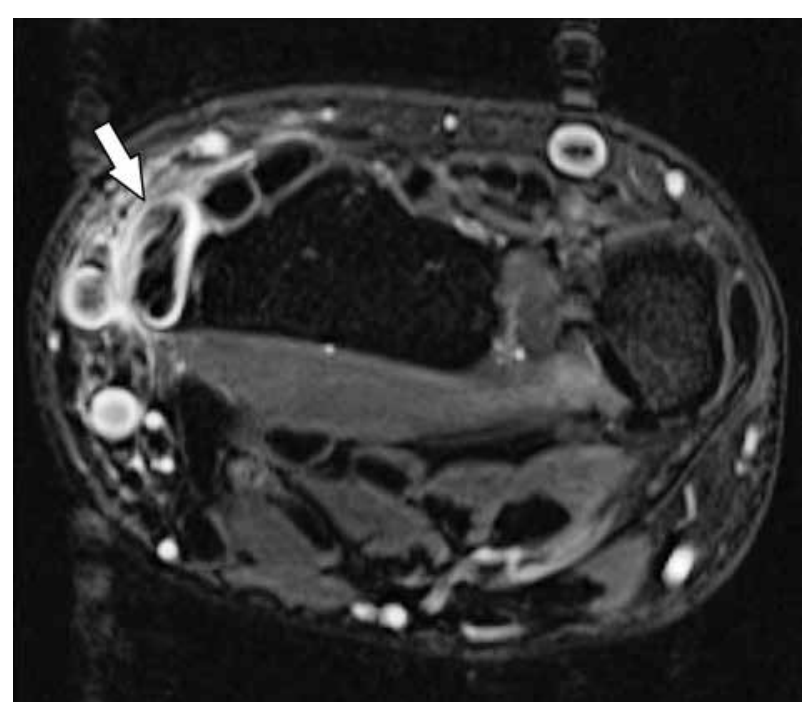

Figure 21. DeQuervain's tenosynovitis. 37-year-old female with radial-sided wrist pain and clicking. Axial T2W fat-suppressed magnetic resonance image shows thickening and signal heterogeneity of the first extensor compartment tendons consistent with tendinosis and distension of the tendon sheath (arrow) compatible with DeQuervain's tenosynovitis

ing over Lister's tubercle and occasionally focal crepitus with movement of the thumb. The condition is most commonly post-traumatic but can also be seen with various rheumatologic and degenerative diseases [26,57].

\section{Entrapment neuropathies}

Entrapment neuropathies are peripheral neuropathies characterised by pain and/or loss of function secondary to nerve compression. 

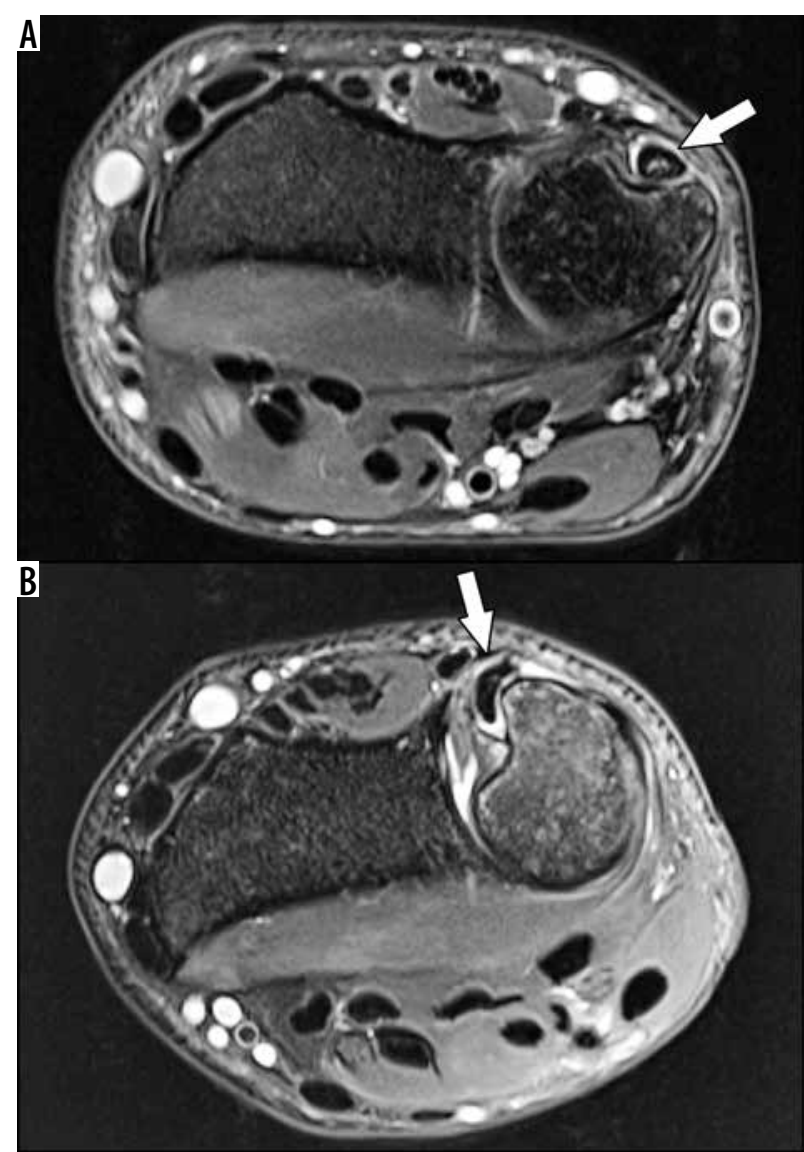

Figure 22. Extensor carpi ulnaris (ECU) interstitial tear. 22-year-old male professional baseball player with left wrist pain and instability after injury 12 weeks prior. A) Axial T2W fat-suppressed (FS) magnetic resonance (MR) image of the wrist at the level of the ulnar groove with the wrist in neutral position shows increased signal in the ECU tendon with a small tenosynovial effusion (arrow). B) Axial T2W FS MR image of the wrist at the level of the ulnar groove with the wrist in supination shows an abnormal boomerang shaped morphology of the ECU tendon, which is draped around the ulnar margin of the groove (arrow). At surgery there was an interstitial tendon tear with insufficiency of the overlying retinaculum that allowed pathologic tendon subluxation

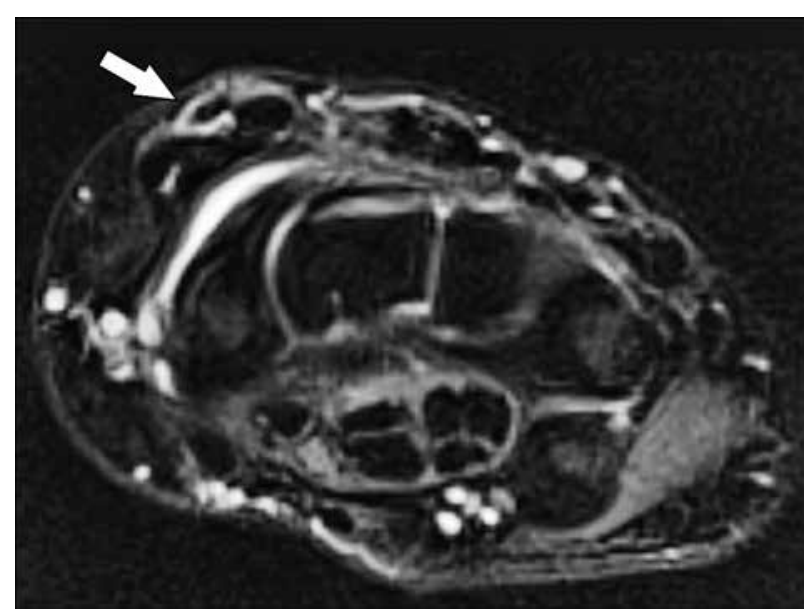

Figure 23. Distal Intersection (Decussation) Syndrome. 69-year-old female with dorsal wrist pain. Axial T2W fat-suppressed magnetic resonance image demonstrates mild peritendinous oedema of the extensor pollicis longus tendon and mild tenosynovitis (third compartment) (arrow) as it crosses over the second extensor tendons at the level of the carpus
Carpal tunnel syndrome is the most common nerve entrapment in the upper extremity. It can be idiopathic or related to various causes of nerve compression, including lunate dislocation, haematoma, or flexor tendinopathy/tenosynovitis related to rheumatologic disorders. Additional aetiologies include diabetes, hypothyroidism, pregnancy, obesity, amyloid deposition, osteoarthritis of the first carpometacarpal (CMC) joint, or space-occupying lesions along the nerve. Clinical symptoms include pain, tingling, and numbness in the thumb, and index and middle fingers. Physical exam findings include atrophy and weakness of the thenar musculature with impaired sensation extending from the thumb to the lateral fourth fingers and with preserved sensation over the thenar eminence. A positive Tinel test, or tingling sensation in the distribution of the nerve elicited by light percussion over the nerve, is an additional sign that supports diagnosis of the disease. MRI findings demonstrate increased signal within the median nerve, enlargement of the nerve at the level of the pisiform, and flattening of the nerve at the level of the hook of hamate (Figure 24). Cross-sectional area (CSA) measurement of the median nerve, including absolute measurements at various points within, proximal and distal to the carpal tunnel, and ratio measurements of CSA in the distal forearm and within the carpal tunnel, can be sensitive in detecting cases of carpal tunnel syndrome. On ultrasound normal median nerve CSA has been reported between 9 and $11 \mathrm{~mm}^{2}$. Although ultrasound measurements have been well-assessed in the literature, MRI measurements are less established. MRI measurements of the median nerve CSA are reportedly routinely larger than those on ultrasound and similar to those measured on cross-sectional cadaveric specimens [58]. A recent work by $\mathrm{Ng}$ et al. has suggested a CSA $>15 \mathrm{~mm}^{2}$ either proximal or distal to the carpal tunnel as a cut-off for detecting carpal tunnel syndrome, and CSA $>19 \mathrm{~mm}^{2}$ as a cut-off for severe carpal tunnel syndrome [59]. Additionally, on axial imaging of the wrist a ratio measurement between the long axis of the median nerve seen in cross section and its short axis at the carpal tunnel inlet of greater than 3:1 may also help to detect cases of carpal tunnel syndrome suggested on nerve conduction testing [60]. Other secondary MRI findings include thickening and bowing of the flexor retinaculum, thenar muscle oedema, and/or atrophy and reactive hypertrophy of the lumbrical muscles. Postcontrast imaging shows enhancement of oedematous nerves and reduced enhancement in ischaemic nerves [26,61].

Complications related to carpal tunnel release are estimated at between 3 and 19\%, and surgical re-exploration is necessary in up to $12 \%$ of cases [61]. Approximately $1-25 \%$ of patients have recurrence of symptoms after surgery [61]. Common causes of recurrent carpal tunnel syndrome include incomplete resection/regrowth of the flexor retinaculum, flexor tenosynovitis, fibrous proliferation, iatrogenic median nerve damage, and extrinsic compression, which can be caused by ganglion cysts or accessory muscle bellies. Regrowth of the flexor retinaculum appears on MRI as a well-defined, continuous linear 


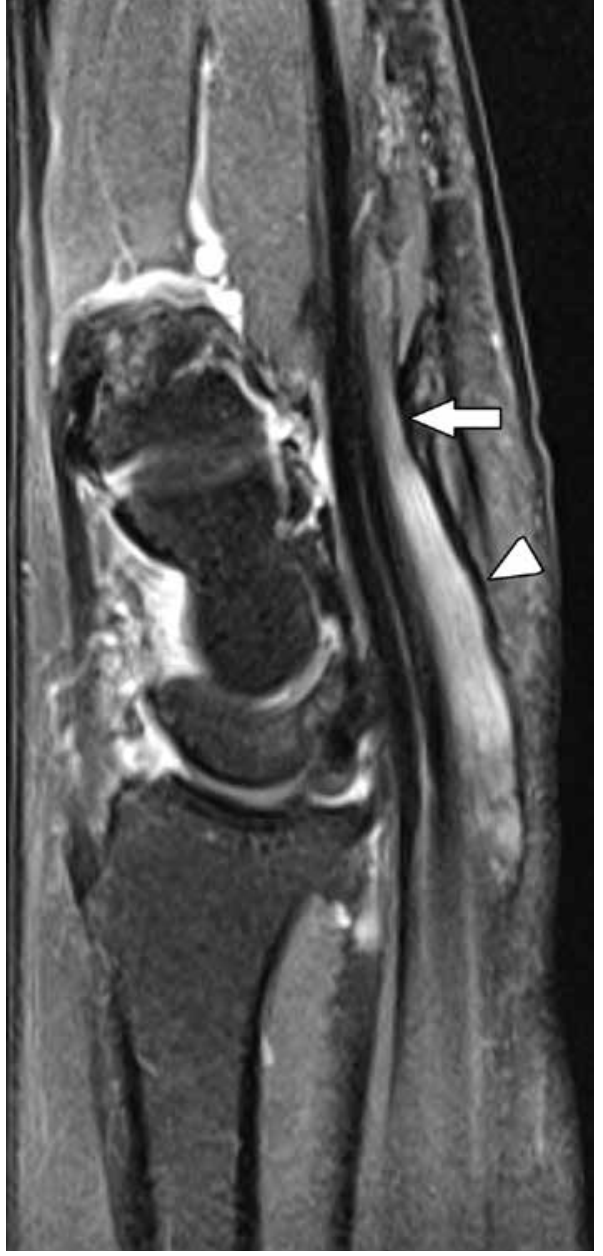

Figure 24. Median nerve compression due to flexor retinacular thickening. 60 -year-old female with chronic numbness in her hand. Sagittal T2W fatsuppressed magnetic resonance image shows flattening and compression of the median nerve (arrow) with thickening of the overlying distal flexor retinaculum, and increased signal in the nerve proximally (arrowhead)

area of low signal intensity superficial to the nerve and thickening of the retinaculum deep to the subcutaneous postoperative scaring. Fibrosis can present as low T1 signal with ill-defined margins within the nerve [61].

Wartenburg syndrome, also known as cheiralgia paresthetica, is a rare syndrome related to isolated pathology of the superficial radial nerve resulting from nerve entrapment between the brachioradialis and extensor carpi radialis longus tendons, which is often due to compression from a watch strap or bracelet. Clinical symptoms include pain over the dorsoradial aspect of the hand [62].

Ulnar nerve compression can occur proximal to Guyon's canal (Type I) or within the canal (Type II) resulting from mass effect related to ganglion cysts, neuromas, or accessory muscles. On rare occasions, ulnar artery pseudoaneurysm, hypertrophy of the flexor carpi ulnaris tendon, osteoarthritis of the pisotriquetral joint, or hamate/pisiform fractures can lead to compression. Clinically, patients present with fourth and fifth finger sensory loss and weakness. Physical examination demonstrates sensory deficits of the little finger and the ulnar half of

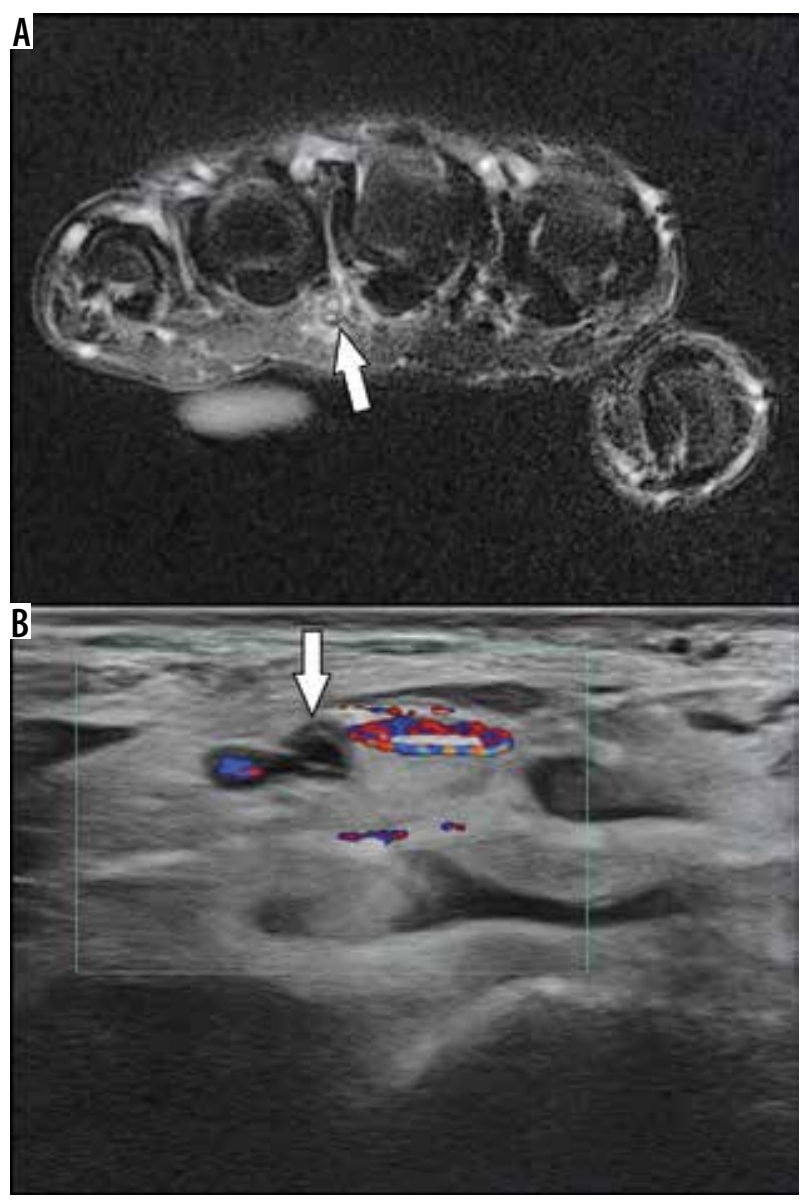

Figure 25. Hypothenar hammer syndrome. 34-year-old male with left ring and middle finger pain beginning a few weeks after doing mechanical work on his car. A) Axial T2W fat-suppressed magnetic resonance image shows increased signal and surrounding soft tissue oedema along the ulnar third interdigital neurovascular bundle (arrow). B) Ultrasound colour Doppler short-axis image shows thrombosis of the ulnar artery more proximally at the level of the wrist (arrow)

the ring finger, as well as a "claw" deformity of the ring and little fingers secondary to intrinsic muscle weakness. MRI findings of Guyon's canal syndrome demonstrate a spaceoccupying lesion along the course of the nerve with an oedematous ulnar nerve proximal to the canal, flattening of the nerve within the canal, and increased signal intensity of the nerve on fluid-sensitive sequences (Figure 2). Secondary signs include muscle oedema and enhancement of the hypothenar muscles, third and fourth lumbricals, and interosseous muscles related to denervation $[26,62]$.

A rare cause of ulnar-sided pain is hypothenar hammer syndrome, which is secondary to thrombosis of the ulnar artery overlying the hamate bone as it exits Guyon's canal. The name of the syndrome comes from patients repetitively hammering objects with the hypothenar aspect of their palms, which causes trauma to the underlying ulnar artery. This results in intimal and medial wall degeneration and thrombosis with distal emboli that can occlude the distal digital arteries and symptomatic ischaemia [63]. MRI can demonstrate an abnormal flow void of the ulnar artery to help suggest this diagnosis (Figure 25). 


\section{Infection}

Closed space infection of the flexor tendon sheaths can result in pyogenic flexor tenosynovitis. Increased pressure within the tendon sheath can lead to tendon rupture or necrosis. Alternatively, adhesions can lead to stenosing tenosynovitis with severe restriction of motion. Infection from the thumb and little finger tendon sheaths can spread into the radial and ulnar bursae and communicate with each other, resulting in a horseshoe abscess. Infection can also spread between the radial and ulnar bursae and rupture into the space of Parona, a potential space between the pronator quadratus fascia and FDP tendon sheath. This is significant because it can allow the spread of infection proximally into the forearm [36].

\section{Magnetic resonance imaging of the lesser fingers}

\section{Anatomy}

The lesser digits include digits 2 through 5. Each digit contains a metacarpal, a proximal phalanx, a middle phalanx, and a distal phalanx. The metacarpals articulate proximally with the carpus forming the CMC joints. Distally, the metacarpals articulate with the proximal phalanges, forming the MCP joints. The proximal and middle phalanges in each digit articulate to form the proximal interphalangeal (PIP) joints. The middle and distal phalanges of each digit articulate to form the distal interphalangeal (DIP) joints.

The primary stabilising ligaments around the MCP and interphalangeal (IP) joints of the lesser digits are the radial and ulnar collateral ligaments, along with the volar plate, which is a fibrocartilaginous thickening of the joint capsule. The collateral ligaments are composed of proper and accessory ligaments. Each volar plate attaches to the collateral ligaments, and to the sagittal bands and deep palmar transverse ligaments at the MCP joint [64].

The extensor mechanism of the lesser fingers is formed by a combination of extrinsic and intrinsic tendons (Figure 26). The extrinsic tendons originate in the forearm, are innervated by the posterior interosseous nerve, and are composed of the extensor digitorum communis (EDC), the extensor indicis proprius (EIP), and the extensor digiti minimi (EDM) [64]. The EDC and EIP tendons travel through the fourth extensor compartment, while the EDM tendon travels through the fifth extensor compartment. A distinct EDC tendon to the fifth digit is only present in approximately half of the population [65]. The EIP tendon most commonly inserts onto the EDC tendon in the second digit, while the EDM tendon travels to the fifth digit. The extensor muscles may either be extrinsic or intrinsic. There is anatomic variation of the extrinsic tendons, which serve to extend the MCP, PIP, and DIP joints of the lesser fingers. The intrinsic extensor tendons are primarily formed by the lumbrical and interosseous muscles. The exception is the abductor digiti minimi, which forms the ulnar lateral band of the fifth digit [65]. The intrinsic tendons continue into the digits as medial and lateral bands.

Between the MCP and PIP joints in each digit, the extensor tendon trifurcates into a central slip and radial and ulnar lateral slips. The central slip inserts onto the dorsal base of the middle phalanx. The lateral slips combine with their respective lumbrical bands distal to the PIP joint to form the conjoined tendons. These conjoined tendons merge in the region of the DIP joint and insert as a terminal tendon at the dorsal base of the distal phalanx (Figure 26). The distal conjoined tendons are connected by the triangular aponeurosis.

The extensor hood is located primarily over the dorsal aspect of the proximal phlangeal base in each lesser digit and serves to stabilise the extensor tendon from abnormal transverse movement. Additionally, the radial and ulnar sagittal bands are located at the MCP joint level in each lesser digit (Figure 26 and 27). They extend around the joint from the extensor hood to the volar plate and serve to stabilise the extensor tendon at the MCP joint level.

The FDS and the FDP tendons form the flexor mechanism of the lesser digits. The FDS muscle originates in the forearm, is innervated by the median nerve, and flexes the joints in the wrist, the MCP, and the PIP joints. The FDP muscle also originates in the forearm, is innervated by the median and ulnar nerves, and flexes the joints in the wrist, the MCP, and IP joints [65]. The FDS tendon is located superficial to the FDP tendon in each lesser digit until the level of the proximal phalanx. The FDS tendon splits at the base of the proximal phalanx of each lesser digit into two distinct tendon slips; it subsequently passes around and eventually deep to the FDP tendon at its insertion onto the volar plate of the PIP joint and at the volar base of the middle phalanx (Figure 28). Just proximal to the FDS insertion, fibres from each tendon slip extend to the other, which is known as Camper's chiasm [27]. The splitting of the FDS tendon provides a passage for the FDP tendon to continue distally within the digit and insert onto the volar plate of the DIP joint and the volar distal phalangeal base.

The flexor tendons are encompassed by digital sheaths from the palm to the DIP joints. Nutrients to the flexor tendons are provided by the vincula breve and longum, which are not typically visualised on routine MRI [65]. Focal areas of digital sheath thickening form a pulley system that serves to secure the flexor tendon to the adjacent bone during finger movement [27]. Although the three cruciform (C) pulleys are poorly visualised on MRI, the five annular (A) pulleys are commonly seen on MRI, particularly on the axial plane, and are numbered according to location. The A1, A3, and A5 pulleys are located at the level of the MCP, PIP, and DIP joints, respectively, and attach to the volar plates. The A2 pulley attaches to the periosteum of the shaft of the proximal phalanx, and the A4 pulley attaches to the periosteum of the shaft of the middle phalanx (Figure 28) [65]. 


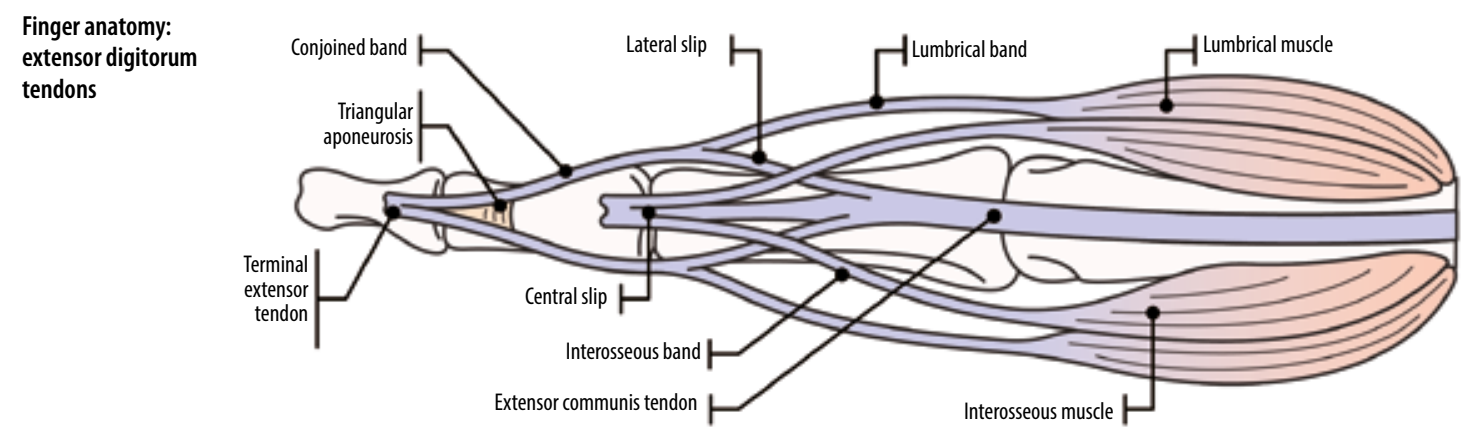

Finger anatomy:
extensor hood ligaments

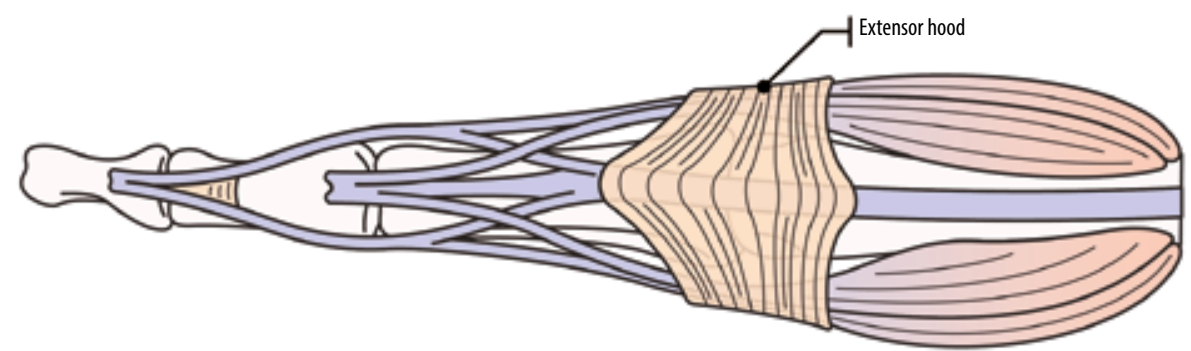

Finger anatomy: extensor sagittal band

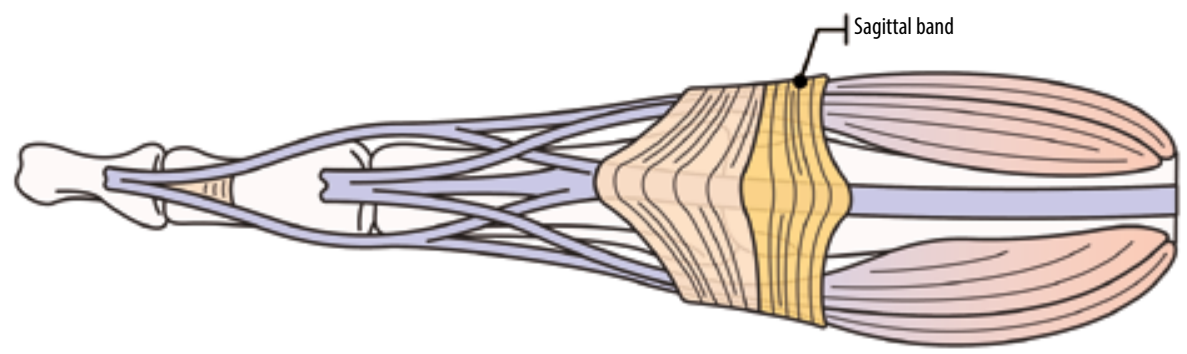

Figure 26. Illustrated diagram of the extensor mechanism of the finger including the tendons, extensor hood ligaments, and extensor sagittal bands

\section{Internal derangement of the lesser fingers}

\section{Bones and joints}

The bones and joints are typically evaluated for contusion, fracture, malalignment, dislocation, or joint effusion in the setting of injury. For fractures involving the articular surface, the amount of involvement should be quantified because it can be an indicator of joint stability. Any fracture displacement should also be described, including the size of any bony fragments. The joints are also evaluated for cartilage integrity and for any evidence of a degenerative or inflammatory arthropathy. Radiographs are typically useful for aiding in evaluation. Additionally, the soft tissues of the lesser digits are evaluated for the presence of haematoma, fluid collection, or oedema in the setting of injury.

\section{Ligaments and volar plate}

On MRI, oedema surrounding the ligaments and volar plates typically indicates acute injury of that structure. Additionally, the structures are evaluated for the pres- ence of partial or full-thickness tearing. The location of any tears should be described, along with any osseous/ periosteal involvement. Ligamentous injuries most commonly involve the PIP joint, and dorsal dislocations can result in volar plate tears. Any imaging evidence of ligamentous/volar plate malpositioning or trapping should be described in the report to aid clinical management.

\section{Extensor mechanism}

Extensor mechanism injuries are commonly classified according to anatomic location. The Verdan system is most commonly used, which includes eight zones (Figure 20) [66]. The odd-numbered zones are located at the joint levels. Injury location, percentage of tendon involvement and any adjacent osseous or soft tissue involvement should be described with detail in the report.

Zone 1 injuries are the most common and are usually closed. They occur at the level of the terminal tendon and frequently arise when the finger is extended and there is forceful flexion of the DIP joint resulting in an avulsion injury, known as a mallet finger. Osseous avulsion from 
A Metacarpophalangeal joint in cross-section

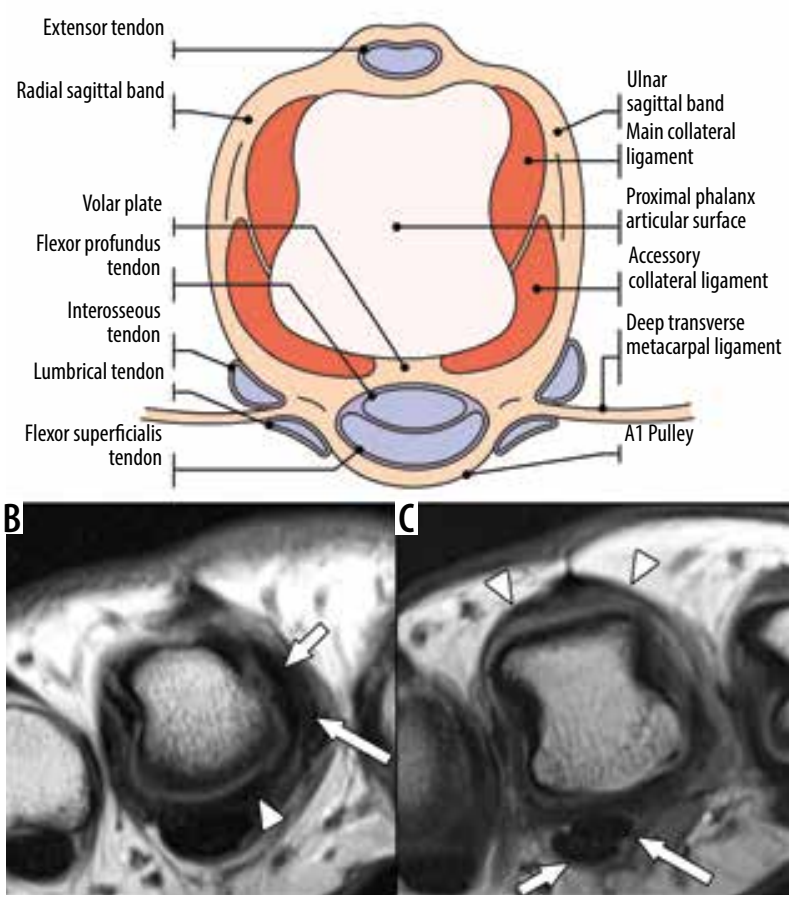

Figure 27. Normal anatomy of the finger at the metacarpophalangeal (MCP) joint. A) Cross-sectional diagram of one of the lesser fingers at the level of the metacarpal head and MCP joint illustrates the positioning of the volar plate, collateral ligaments and flexor and extensor tendons. B) Axial proton density (PD) non-fat suppressed (NFS) magnetic resonance (MR) image of the finger at the level of the metacarpal head in a 42-year-old female shows the fibrocartilaginous thickening of the volar plate (arrowhead). On the radial side the main (shorter arrow) and accessory collateral ligaments (longer arrow) are depicted. C) On a slightly proximal axial PD NFS MR image in the same finger the centrally located extensor digitorum communis tendon with the adjacent radial and ulnar sagittal bands can be seen (arrowheads). On the palmar side the flexor digitorum superficialis and flexor digitorum profundus tendons are also visible (shorter arrow and longer arrow, respectively)

the dorsal base of the distal phalanx can occur. Zone 1 injuries are typically treated by splinting the finger with the DIP joint in extension.

Zone 2 injuries are usually due to a laceration and occur at the level of the middle phalanx. These injuries commonly involve the conjoined tendon(s) and/or triangular ligament. Injury to one of the conjoined tendons is usually managed conservatively. Surgical repair is usually indicated if the injury involves the triangular ligament and/or both tendons.

Zone 3 injuries can be open or closed and occur at the PIP joint level. Closed injuries are usually caused by a blow to the dorsum of the finger, forceful flexion of the PIP joint, or volar joint dislocation. These injuries can involve the distal central tendon slip with possible osseous avulsion of the dorsal middle phalangeal base, but they can also involve the lateral slips. Many injuries can be treated conservatively by splinting with the PIP joint in extension. However, open injuries, displaced osseous avulsion fragments, and instability may necessitate surgical intervention.

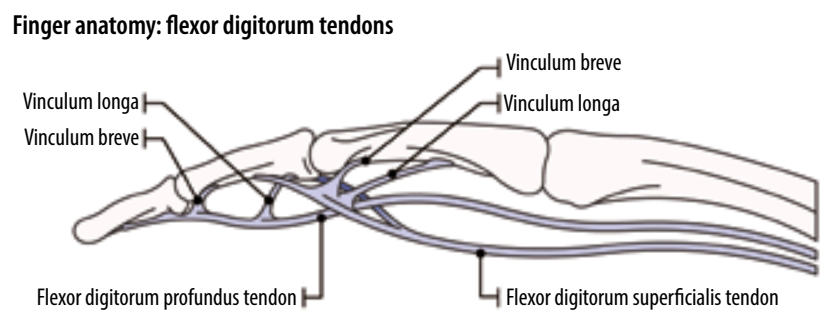

Finger anatomy: flexor pulley ligaments

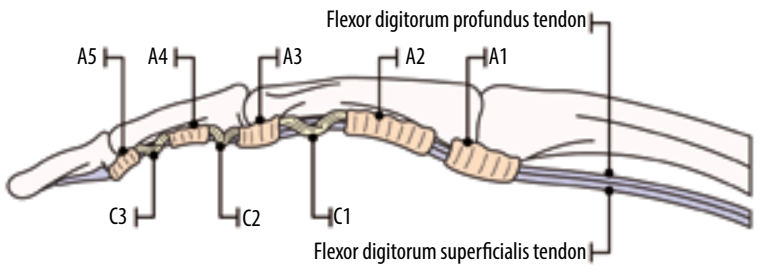

Finger anatomy: flexor collateral ligaments

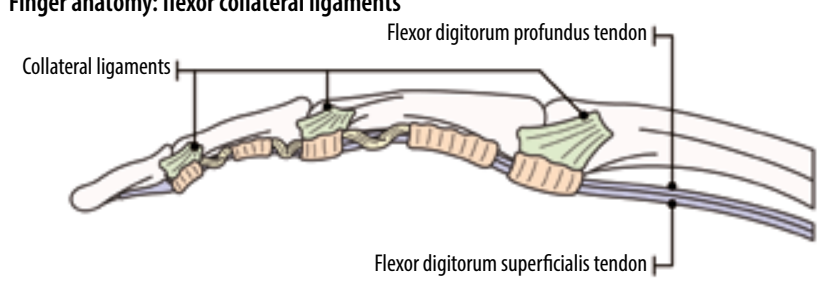

Figure 28. Illustrated diagram of the flexor anatomy of the finger including the tendons and annular and cruciate pulleys

Zone 4 injuries at the level of the proximal phalanx often result from lacerations and can involve the central slip, lateral slips, medial and lateral lumbrical bands, and extensor hood. Most injuries in this zone are partial tears without significant retraction since the extensor mechanism in this region is broad. Injuries in this zone are usually treated conservatively with splinting with the PIP joint in extension. Higher grade injuries usually require surgical intervention.

Zone 5 injuries at the level of the MCP joint commonly occur at the index finger MCP joint with flexion. These injuries are usually due to a biting mechanism and involve the extensor tendon with either a complete or partial tear. The sagittal band and joint capsule can also be involved (Figure 29). Oblique injuries involving the sagittal band can be

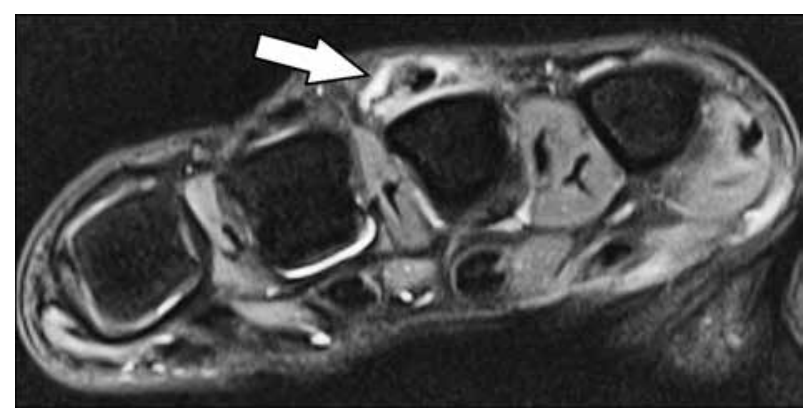

Figure 29. Focal extensor digitorum communis (EDC) tendon tear at extensor tendon zone 5 with tearing of the ulnar sagittal band. Axial T2W fat-suppressed magnetic resonance image of a 37-year-old female with pain and swelling dorsal to the left third metacarpophalangeal joint shows partial tearing of the EDC tendon with tendon thickening consistent with tendinopathy. This is associated with a focal tear of the ulnar sagittal band as it attaches onto the tendon (arrow) 
open but are more commonly closed; and can involve subluxation or dislocation of the extensor tendon, often resulting in a snapping sensation for the patient. There is concern for infection with open injuries, including septic arthritis if there is involvement of the joint capsule. Surgical repair is frequently required for zone 5 injuries.

Zone 6 injuries in the dorsum of the hand are usually due to laceration. The extensor tendons are highly superficial in this region. Multiple extensor tendons can be involved with a single injury. Clinical exam may not demonstrate a significant loss of extension with single tendon or partial tendon lacerations due to connections between the tendons. Surgical repair is required.

\section{Flexor mechanism}

MRI is useful for evaluating flexor tendon injuries. $\mathrm{T} 2 \mathrm{~W}$ sequences typically demonstrate fluid signal in the region of a recent tear [27]. Important imaging findings include whether the tear is partial-thickness (with an estimate of the cross-sectional percentage of tendon involvement) or full-thickness. Location of the tear and any tendinous retraction should be clearly described, in addition to the presence of any osseous avulsions and any displacement of the bony fragment.

Flexor tendon injuries have been classified into five anatomic zones by Kleinert et al. and Verdan (Figure 20) $[67,68]$. Flexor tendon injuries are more commonly open, and often due to laceration. Less commonly, they may be closed and related to avulsion. The most common type of closed avulsion injury is a tear of the distal FDP tendon. This usually occurs due to forced hyperextension of the DIP joint during active flexion, in activities such as football or rugby, and most often involves the ring finger (Jersey finger). Leddy and Packer initially classified these injuries into three predominant types [69], with additional types subsequently described. Type I injuries result when the tendon is retracted into the palm. Type II injuries occur when the tendon is retracted to the PIP joint level. Type III injuries are present when there is an avulsed bone fragment, with the fragment held in place by the A4 pulley; type IV injuries occur when the bony fragment is present, but the tendon has been avulsed from the fragment [64]. The majority of these injuries require surgical repair, and type I injuries are often urgent due to blood supply compromise [27]. Isolated FDS avulsions are rare, and FDS injuries usually occur in conjunction with FDP injuries.

Flexor tendon laceration commonly occurs in the mid substance rather than at the insertion [70] and is classified according to its location in the zones described by Kleinert et al. and Verdan (Figure 30). Injuries in zones 2 through 5 can involve the neurovascular bundle and require emergency surgery [27]. Zone 2 injuries are the most common and are associated with the highest rate of complications. Although traditionally zone 2 injuries were not repaired surgically due to the high rate of complica-

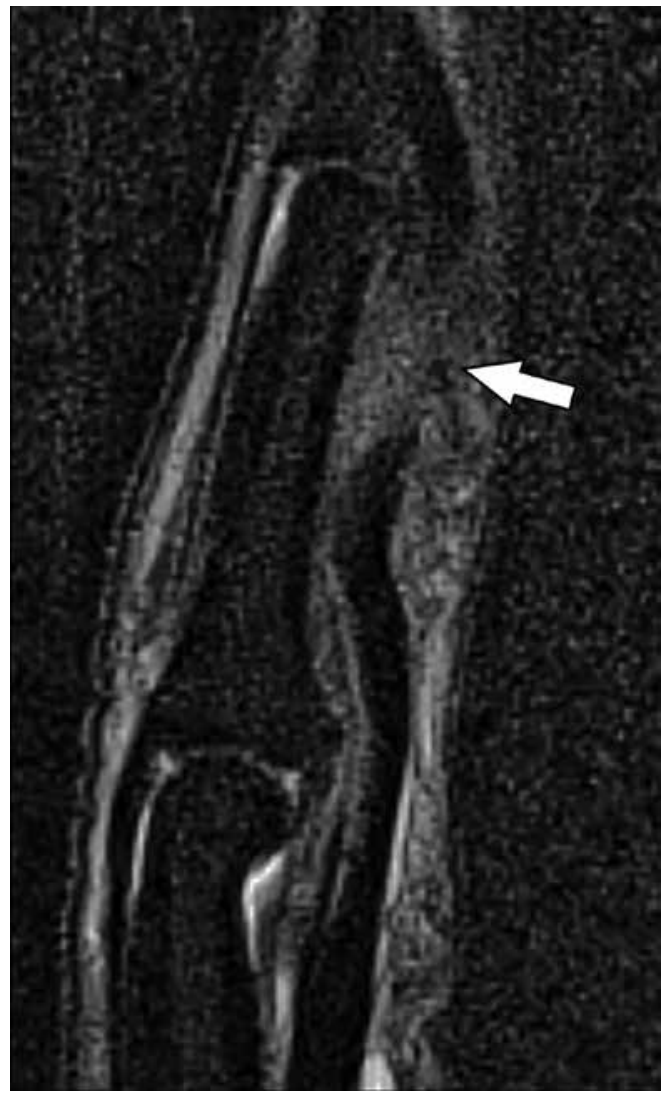

Figure 30. Distal flexor digitorum profundus (FDP) tendon laceration in flexor zone 1. Sagittal T2W magnetic resonance fat-suppressed image of the left middle finger in a 23-year-old male who suffered a laceration demonstrates the complete FDP tendon tear distal to the flexor digitorum superficialis insertion (arrow) with a $1.1 \mathrm{~cm}$ tendon gap

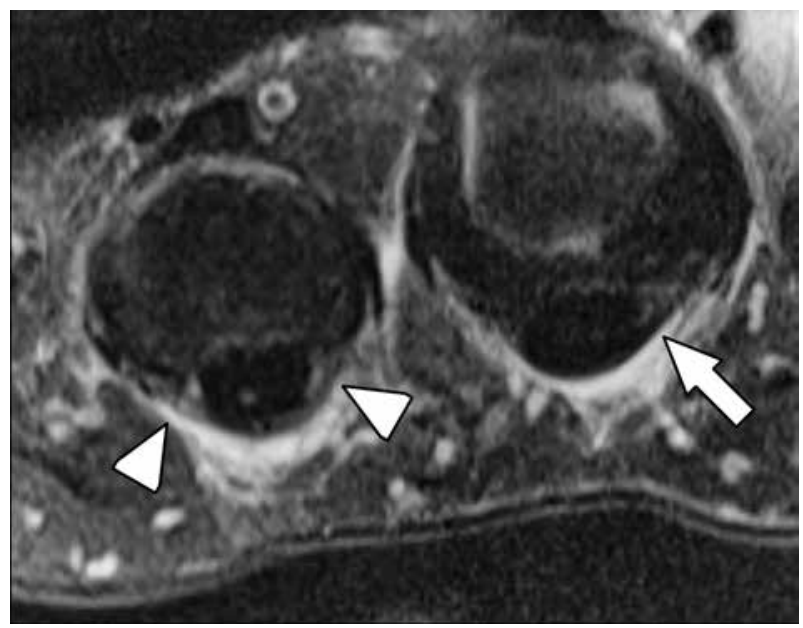

Figure 31. Low-grade A1 flexor tendon pulley injury. Axial T2W fat-suppressed magnetic resonance image of the third and fourth metacarpal heads in a 59-year-old female who sustained a low energy trauma to the middle and ring fingers shows mild thickening, intermediate signal, and surrounding oedema of the ring finger A1 pulley (arrowheads). There is mild oedema around the middle finger A1 pulley. However, the pulley fibres appear intact, uniform in thickness, and low in signal (arrow)

tions, these injuries are currently repaired surgically due to advances in understanding of these injuries and surgical technique. Surgical repair for these injuries ideally is performed within 24 hours in current practice [27]. 
Injuries to the annular pulleys are best visualised on axial imaging (Figure 31). Rock climbing is a common cause of pulley injury, most frequently involving the A2 pulley of the ring finger [27]. Tears can either be partial or complete and can involve either a single pulley or multiple pulleys. A consequence of pulley tearing is bowstringing of the flexor tendon, which is variable depending on the number of pulleys torn and the positioning of the finger [27]. Comparison with the adjacent finger flexor mechanism can be helpful when trying to assess for subtle bowstringing on MRI. Fluid signal around the pulleys is usually seen in the setting of acute injury. Klauser et al. examined the degree of displacement of the flexor tendon from the volar surface of the proximal phalanx (the tendon-to-phalanx [TP] distance) at rest and with forced finger flexion on both MRI and ultrasound in elite rock climbers. They observed that a TP distance of more than $1.0 \mathrm{~mm}$ was a sign of pulley insufficiency and at least partial tearing and an increase in palmar tendon displacement of at least $1.0 \mathrm{~mm}$ with forced finger flexion compared with rest was also a sign of at least partial tearing. With forced finger flexion, a TP distance of more than $2.5 \mathrm{~mm}$ may be helpful in diagnosing a complete tear of either the A2 or A4 pulleys [71].

\section{Magnetic resonance imaging of the thumb}

\section{Thumb anatomy}

The thumb is composed of the proximal and distal phalanges, the first metacarpal and CMC joint, and trapezium with four basal joints, including its articulations with the scaphoid, trapezoid, and first and second metacarpal bases.

The first CMC joint is a biconcave, saddle-shaped joint between the trapezium and the first metacarpal base that allows significant range of motion, including flexion with medial rotation, extension with lateral rotation, abduction/ adduction, and circumduction (rotational movement) [72]. It is statically stabilised by a number of ligaments (Figure 32). Historically, the most important of these was felt to be the anterior oblique ligament (AOL or beak ligament), which is a palmar structure traveling between the palmar tubercle of the trapezium and the ulnar margin of the first metacarpal base. It contains deep and superficial bands and is a chief restraint to dorsal radial subluxation. The more dorsal ligaments include the posterior oblique ligament, dorsoradial ligament (DRL), intermetacarpal ligament, and UCL (Figure 33). Recent evidence suggests that these ligaments, particularly the DRL, which is the thickest and strongest of the dorsal ligaments, also play a critical role in preventing dorsal metacarpal base subluxation [73].

The first MCP joint is a diarthrodial joint that has features of both a condyloid (ellipsoid) joint and a hinged (ginglymus) joint, which allows a greater range of motion than a typical hinge joint. The MCP joint capsule is partially composed of fibrocartilage that is thickened dorsally and volarly
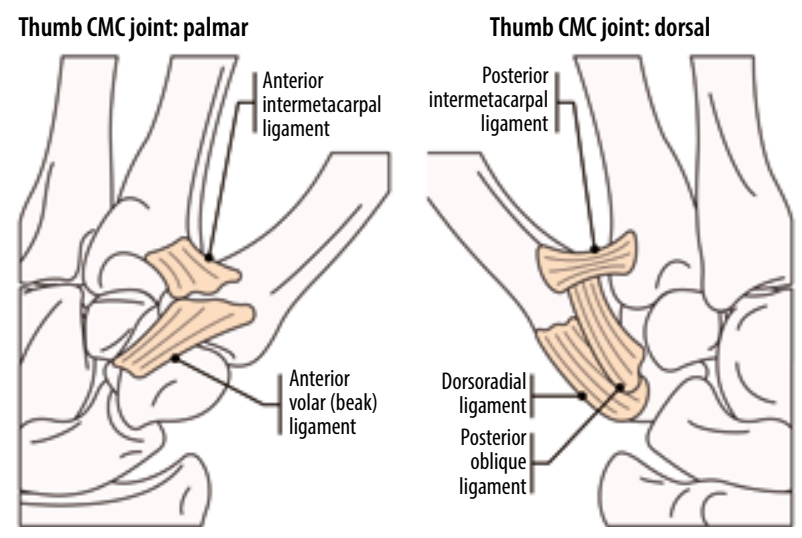

Figure 32. Illustrated schematic of the palmar and dorsal ligaments of the first carpometacarpal joint

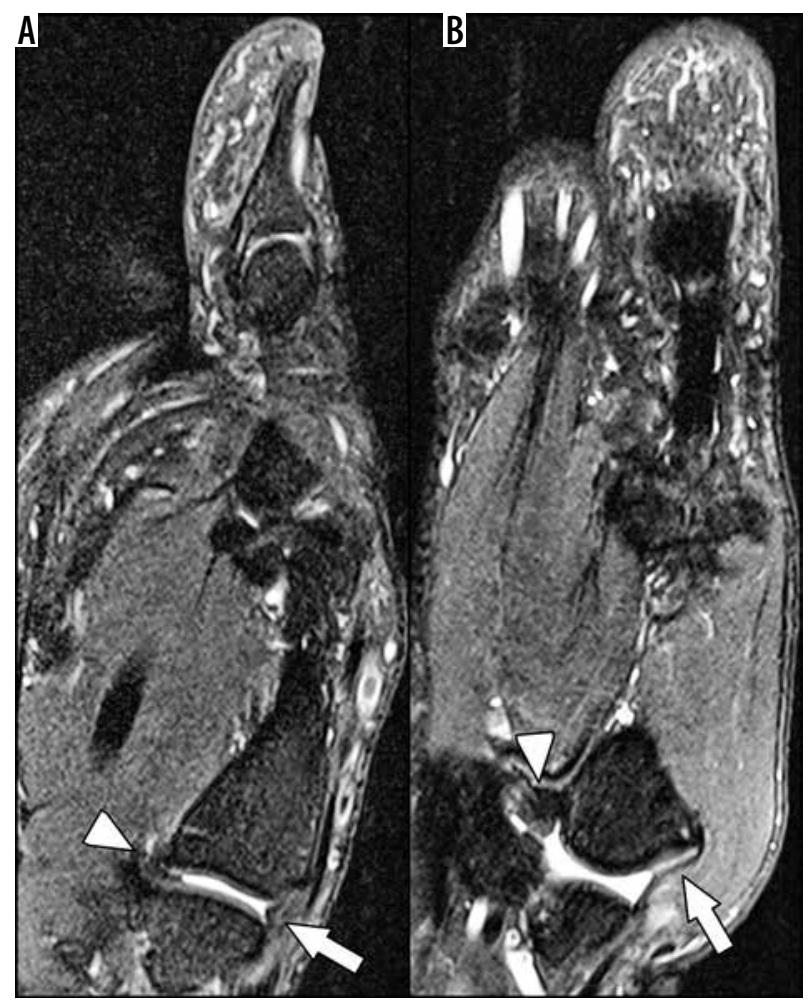

Figure 33. Normal first carpometacarpal ligaments in a 36-year-old male. A) Sagittal T2W fat-suppressed (FS) magnetic resonance (MR) image of the thumb shows the normal anterior oblique ligament and dorsoradial ligament (arrowhead and arrow, respectively), which help to stabilise the thumb and prevent dorsal/volar subluxation. B) Coronal T2W FS MR image shows the normal intermetacarpal (arrowhead) and ulnar collateral ligaments (arrow)

and forms the dorsal and volar plates, respectively. On MRI, the normal dorsal and volar plates are best seen on sagittal imaging as triangular-shaped low signal intensity structures. There are usually two palmar sesamoid bones surrounded by the volar plate, which attaches to the metacarpal head and proximal phalangeal base and helps stabilise the flexor pollicis longus tendon. In addition, there is commonly a small joint recess between the proximal phalangeal base and plate [74]. The radial and ulnar collateral ligaments resist varus and valgus angular forces, respectively. They are composed 


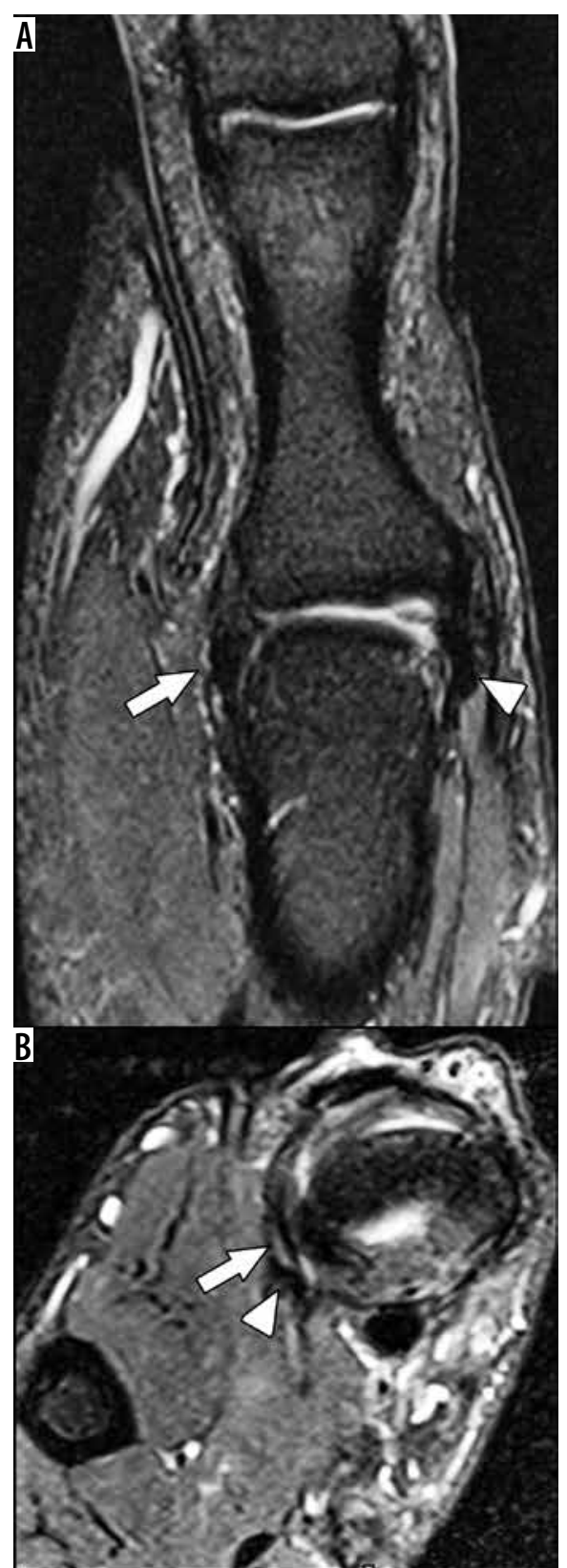

Figure 34. Normal metacarpophalangeal (MCP) ligaments of the thumb. A) Coronal T2W fat-suppressed (FS) magnetic resonance (MR) image of the thumb at the MCP joint in a 38-year-old male shows the normal appearance of the ulnar collateral ligament (UCL, arrow) and radial collateral ligament (arrowhead). The ligaments are smooth and of low signal as they attach to the metacarpal and the proximal phalanx, and there is no surrounding soft tissue or bone marrow oedema. Also note normal collateral ligaments of the thumb interphalangeal joint. B) Axial T2W FS MR image of the thumb in the same patient shows the positioning of the main and accessory bands of the UCL (arrow and arrowhead, respectively)

of a proper ligament, which courses from the dorsal metacarpal head to the volar proximal phalangeal base, and an accessory ligament, which is more volar and courses from the metacarpal head to the volar plate and sesamoid bones (Figure 34). The proper ligament is taut in flexion and lax in extension. Importantly, the adductor aponeurosis arises from the adductor pollicis and courses superficially to the UCL to attach onto the proximal shaft of the proximal phalanx [75].
A Thumb anatomy dorsal view: extensor tendons

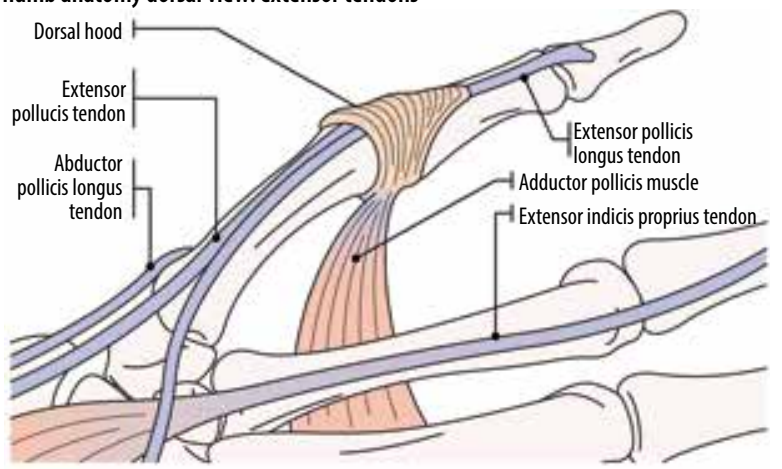

B Thumb anatomy palmar view: flexor pulleys

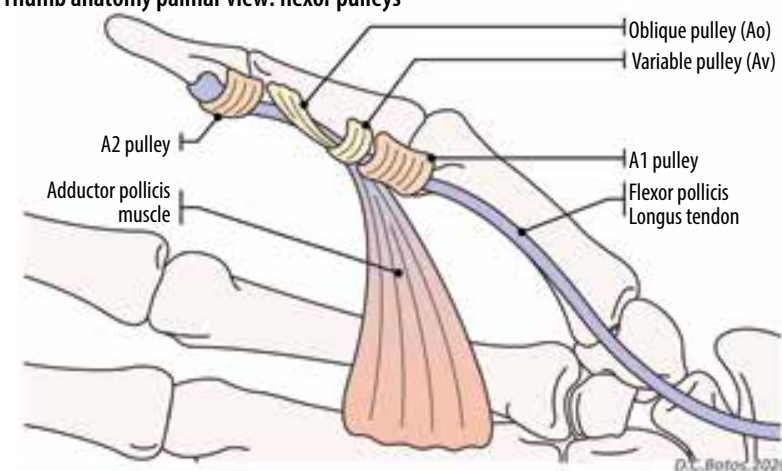

C

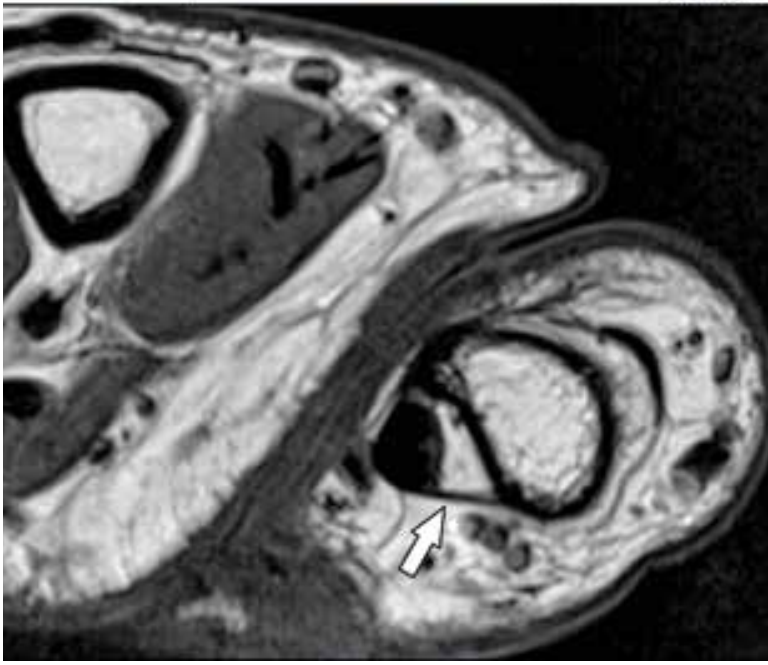

Figure 35. Normal thumb tendons and pulleys. A) Illustration of the dorsum of the thumb extensor tendons shows the attachment sites of the tendons and the extensor apparatus. B) Illustration of the palmar aspect of the thumb shows the flexor pollicis longus tendon pulleys and the adductor muscle. C) Axial T1W non-fat suppressed magnetic resonance image in a 38-year-old male shows the Av pulley at the mid proximal phalangeal shaft level as a thin band of fibrous tissue overlying the flexor pollicis longus tendon. The tendon is slightly ulnarly positioned due to the longer radial limb of this pulley (arrow)

Finally, the IP joint is a true hinge joint, allowing flexion and extension. Similar to the thumb MCP joint, the IP joint has dorsal and volar plates consisting of fibrocartilage [74]. Because it permits the least mobility it is the most stable of these joints and the least commonly injured [72].

The flexor and extensor tendons dynamically stabilise the thumb and contribute to its motion. The flexor pollicis longus tendon originates in the forearm along the volar as- 
pect of the radius and extends through the radial portion of the carpal tunnel to the palmar surface of the thumb between the MCP sesamoid bones overlying the volar plate. It inserts on the volar distal phalangeal base and flexes the IP and MCP joints (Figure 35). The thumb pulleys help to stabilise the tendon and attach it to the volar surfaces of the MCP joint, proximal phalanx, and IP joint [71]. Unlike the lesser fingers, the thumb only has A pulleys; the A1 pulley is located at the MCP joint and the A2 pulley is at the IP joint. An oblique annular pulley (Ao) is located at the level of the mid proximal phalanx. In about $93 \%$ of cadaveric specimens there is also a variable pulley (Av) located between the A1 and Ao pulleys. The radial limb of this pulley is slightly longer, and the tendon is usually positioned slightly ulnar to the midline of the joint [75].

Two wrist extensor compartments serve to abduct and extend the thumb. The first extensor compartment is composed of the abductor pollicis longus tendon, which has a dominant insertion on the radial margin of the first metacarpal bone, and the extensor pollicis brevis tendon, which inserts on the proximal phalangeal base or the dorsal first MCP joint capsule. On the other hand, the third extensor compartment is only composed of the extensor pollicis longus tendon, which inserts on the dorsal distal phalangeal base and allows extension of the IP joint [73]. This tendon is stabilised by the extensor hood at the MCP joint, which has radial and ulnar sagittal bands, and slightly more distal triangular expansions, which extend distally to insert on the distal phalangeal base [75].

\section{Magnetic resonance imaging of thumb internal derangement}

\section{First CMC joint}

Acute ligamentous injuries of the thumb can occur but are much less common than at the MCP joint. The most common internal derangement in this location is osteoarthritis. This is generally due to ligamentous laxity related to repetitive movements such as pinching or a single traumatic event. There is a higher incidence of first CMC osteoarthritis in women, which has been associated with hormonal influences. Radiography is the primary tool for assessing osteoarthritis, but MRI can be useful to look for ligament injuries, such as to the AOL, which can result in joint instability. Furthermore, MRI often detects signs of osteoarthritis earlier than radiography. Finally, MRI can be used to look for additional causes of radial-sided pain, such as DeQuervain's tenosynovitis or radiographically occult fractures. Typical MRI findings include synovial proliferation and effusion, cartilage loss, bone marrow oedema with subchondral cyst-like changes, and marginal osteophytes. The stabilising ligaments may be thickened and intermediate in signal, indicating chronic scarring and degeneration. As with acute ligament injuries elsewhere, MRI findings include thickening and intermediate/

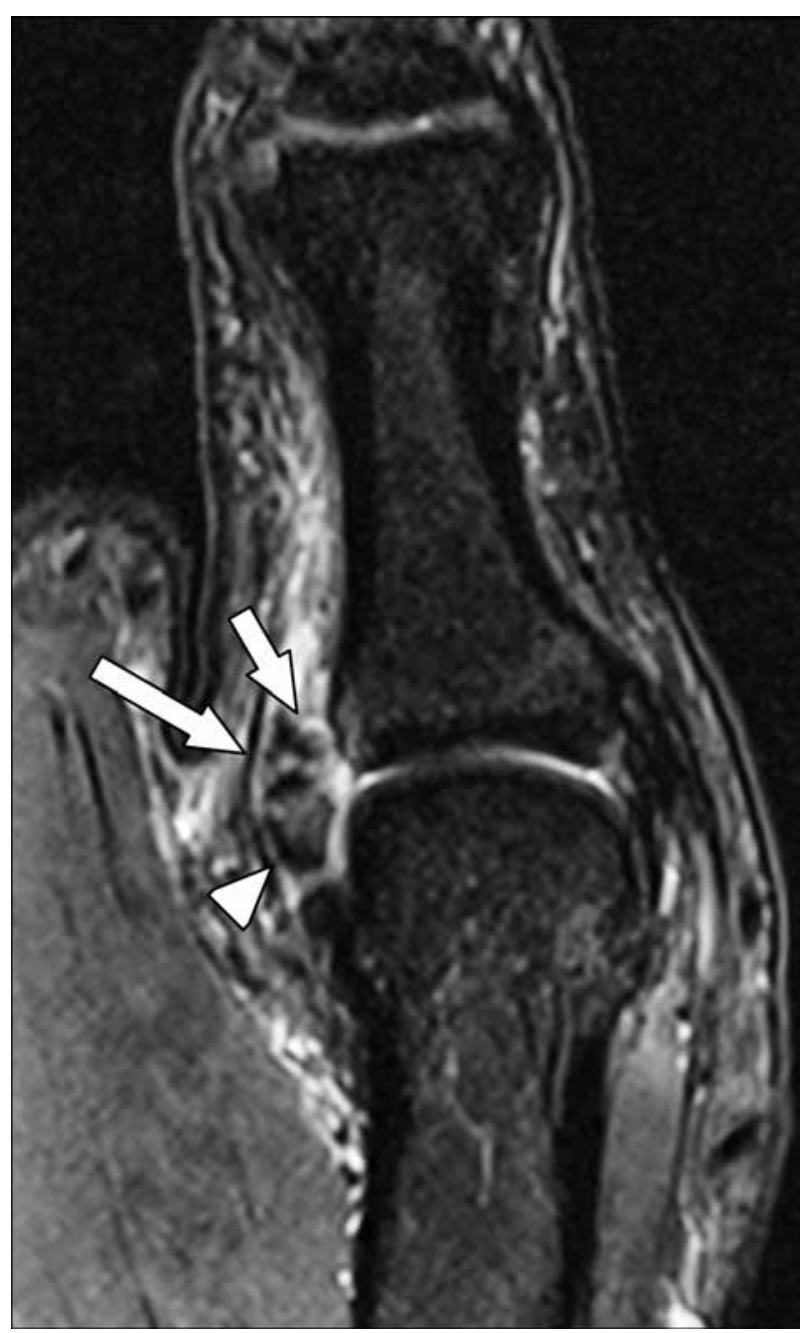

Figure 36. First metacarpophalangeal (MCP) ulnar collateral ligament (UCL) osseous avulsion in a 26-year-old male professional baseball player who injured his thumb while sliding into a base. Coronal T2W fat-suppressed magnetic resonance image of the thumb at the MCP joint depicts an osseous avulsion of the UCL from the proximal phalangeal base (arrowhead) with subtle marrow oedema in the proximal phalangeal base and a fluid gap between the torn ligament and the proximal phalanx (shorter arrow). However, no Stener lesion is seen and the adductor aponeurosis is noted overlying the torn UCL (longer arrow)

increased signal within the ligament with periligamentous oedema, and a fluid signal intensity gap either within the ligament or at its osseous attachments, which indicates a partial or complete tear [76].

\section{First MCP joint}

UCL injuries are commonly related to hyperabduction of the thumb, particularly while grabbing an object such as a ski pole during a fall. As a result, this condition is commonly called skier's thumb. The term gamekeeper thumb has been used synonymously, although it more commonly refers to chronic injuries, while skier's thumb is generally reserved for more acute injuries. UCL tears most often occur distally at the proximal phalangeal attachment and may be associated with osseous avulsions; however, they can be 


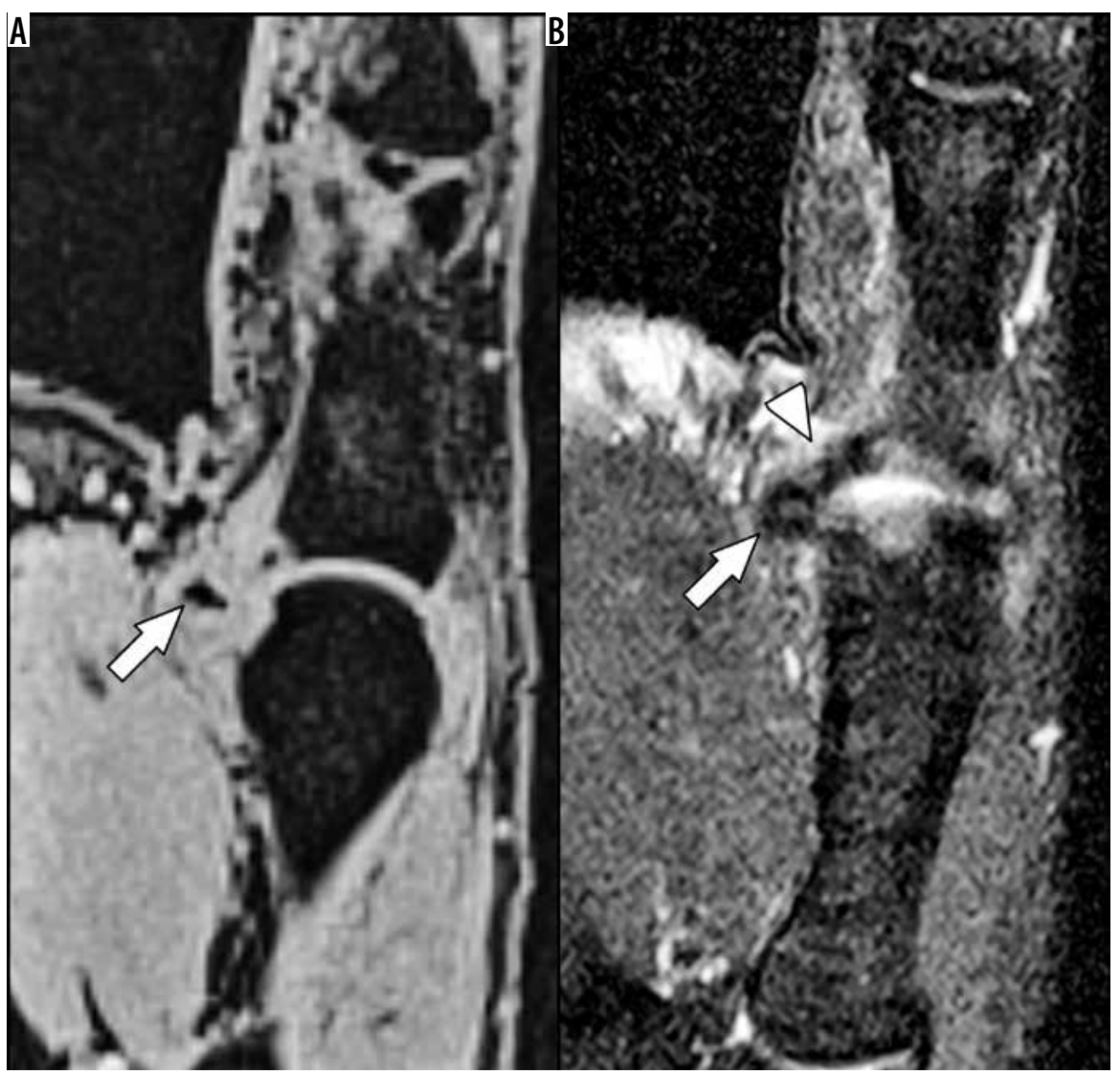

Figure 37. First metacarpophalangeal (MCP) ulnar collateral ligament osseous avulsion with Stener lesion in a 39-year-old female with left thumb valgus injury. A) Coronal T1W fat-suppressed (FS) gradient echo magnetic resonance (MR) image of the MCP joint shows a fracture fragment off the ulnar aspect of the proximal phalangeal base (arrow) that is displaced by $6 \mathrm{~mm}$. This is an indication for surgical repair. B) Coronal T2W FS MR image of the same area shows a Stener lesion with a "yo-yo on the string" appearance. The arrowhead indicates the adductor aponeurosis (string), while the arrow shows the retracted ligament and osseous fragment (yo-yo)

proximal, near the metacarpal head attachment, or in the mid substance [77]. A Stener lesion occurs when the distal UCL tears and the overlying adductor aponeurosis becomes interposed between the UCL stump and the proximal phalangeal base. This prevents healing of the UCL and leads to joint instability and osteoarthritis [13].

MRI is very helpful in diagnosing and characterising UCL injuries. It has been reported that the sensitivity for complete proper UCL tears is $96 \%$, while the specificity is 95\% [78]. Injuries of the UCL can be divided into those that are acute or those that are chronic, and the MRI findings will differ. In acute UCL injuries there is commonly periligamentous oedema seen on fluid-sensitive sequences. The ligament may be thickened, ill-defined, and contain internal intermediate signal. A complete tear is diagnosed when there is a fluid signal gap within the ligament or at the ligament attachment on to the bone, and there may be an associated avulsion fracture with variable degrees of bone marrow oedema (Figure 36) [13]. If the avulsed osseous fragment is displaced by more than $5 \mathrm{~mm}$, or if it involves more than $25 \%$ of the articular surface, surgery is indicated.

In chronic injuries, the soft tissue and marrow oedema usually resolve, and the ligament may appear thickened and irregular, which reflects chronic scarring. There may be a gap between the bone and the ligament stump, and the ligament stump may be retracted and globular. In these cases, the adductor aponeurosis can lie deep to the UCL stump, which forms the classic "yo-yo on a string" appearance described in the Stener lesion (Figure 37). In this finding, the string represents the aponeurosis, while the yo-yo is the thickened ligament stump [79]. Some authors have noted that an intact accessory band or sesamoid-metacarpal ligament may prevent the development of a Stener lesion [80]. MRI may be helpful to assess the integrity of these structures, to determine whether a patient may be prone to developing a Stener lesion [64,74].

RCL injuries are much less common than UCL injuries. Although most injuries are lower grade sprains and can be managed with immobilisation, higher grade partial and complete tears have been shown to contribute to joint instability, chronic ulnar deviation, and MCP osteoarthritis. As a result, these injuries are commonly treated surgically [79]. There are rare reports of a Stener-like lesion involving interposition of the abductor aponeurosis and the RCL, which also requires surgical intervention [81].

\section{Trigger finger/trigger thumb}

Trigger finger/trigger thumb results in a painful click with restricted range of motion. This can produce a locked 


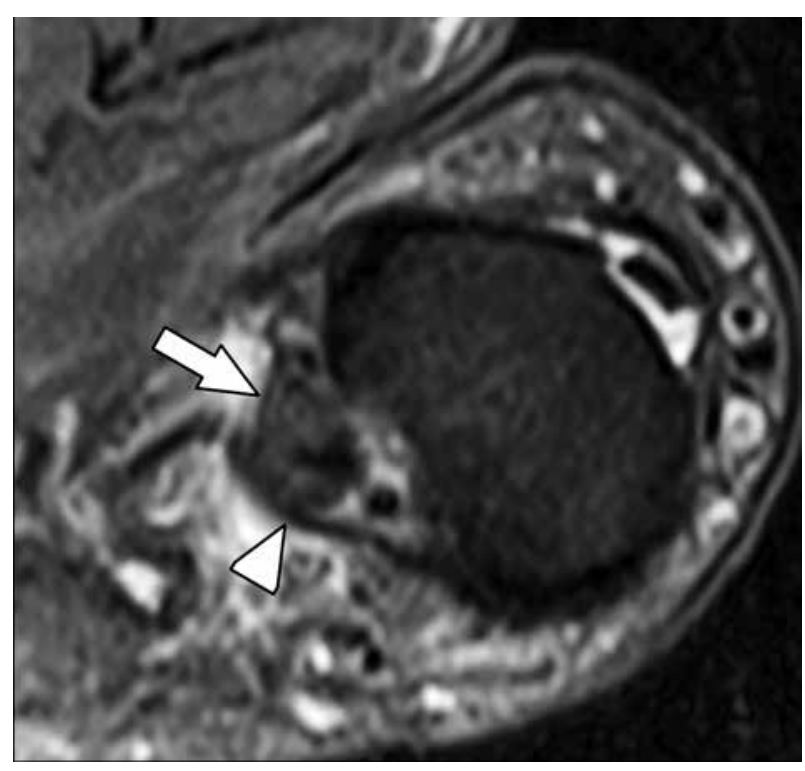

Figure 38. Trigger thumb. 65-year-old male who was experiencing thumb clicking and limited range of motion. Axial T2W fat-suppressed magnetic resonance image of the thumb at the first metacarpophalangeal level shows mild flexor pollicis longus tendinosis with thickening and intermediate intrasubstance signal (arrow). Additionally, the A1 pulley is mildly thickened and intermediate in signal (arrowhead)

digit that must be manually unlocked. It usually occurs in the fifth and sixth decades of life and is more common in diabetic patients. It is caused by flexor tendon and pulley fibrocartilaginous metaplasia, usually the proximal margin of the A1 pulley. MRI may demonstrate tendon thickening and intermediate intrasubstance signal, indicating tendinosis, and there may be thickening of the pulley (Figure 38). When this occurs in the thumb, it may spontaneously resolve. However, surgery is indicated for persistent triggering.

\section{Conclusions}

MRI provides exquisite contrast and spatial resolution to allow accurate, noninvasive assessment of all musculoskeletal tissues of the wrist and hand. In order to generate diagnostic images in a reasonable time, patient positioning, scanner and coil selection, and MRI parameters must be optimised, and imaging should be tailored to best address the clinical question. Newer MRI techniques, such as $3 \mathrm{D}$ volumetric acquisition, offer improvements in image quality while allowing imaging to be done more rapidly.

\section{Conflict of interest}

The authors report no conflict of interest.

\section{References}

1. Johnson D, Stevens KJ, Riley G, et al. Approach to MR imaging of the elbow and wrist: technical aspects and innovation. Magn Reson Imaging Clin N Am 2015; 23: 355-366.

2. Morris CJ, Younan Y, Singer AD, et al. Masses of the hand and wrist, a pictorial review. Clin Imaging 2016; 40: 650-665.

3. Howe BM, Spinner RJ, Felmlee JP, et al. MR imaging of the nerves of the upper extremity: elbow to wrist. Magn Reson Imaging Clin N Am 2015; 23: 469-478.

4. Von Borstel D, Wang M, Small K, et al. High-resolution 3T MR imaging of the triangular fibrocartilage complex. Magn Reson Med Sci 2017; 16: 3-15.

5. Cody ME, Nakamura DT, Small KM, et al. MR imaging of the triangular fibrocartilage complex. Magn Reson Imaging Clin N Am 2015; 23: 393-403.

6. Hobby JL, Tom BD, Bearcroft PW, et al. Magnetic resonance imaging of the wrist: diagnostic performance statistics. Clin Radiol 2001; 56: 50-57.

7. Gold GE, Chen CA, Koo S, et al. Recent advances in MRI of articular cartilage. Am J Roentgenol 2009; 193: 628-638.

8. Chang AL, Yu HJ, von Borstel D, et al Advanced imaging techniques of the wrist. Am J Roentgenol 2017; 209: 497-510.

9. Steinbach LS, Smith DK. MRI of the wrist. Clin Imaging 2000; 24: 298-322.

10. Berquist TH (ed.) MRI of the musculoskeletal system. Lippincott Williams \& Wilkins, Philadeplhia 2012.

11. Morris CJ, Younan Y, Singer AD, et al. Masses of the hand and wrist, a pictorial review. Clin Imaging 2016; 40: 650-665.
12. Østergaard M, Emery P, Conaghan PG, et al. Significant improvement in synovitis, osteitis, and bone erosion following golimumab and methotrexate combination therapy as compared with methotrexate alone: a magnetic resonance imaging study of 318 methotrexate-naive rheumatoid arthritis patients. Arthritis Rheum 2011; 63: 3712-322.

13. de Mooij T, Riester S, Kakar S. Key MR imaging features of common hand surgery conditions. Magn Reson Imaging Clin 2015; 23: 495-510.

14. Schmitt R, Fröhner S, van Schoonhoven J, et al. Idiopathic osteonecrosis of the scaphoid (Preiser's disease) - MRI gives new insights into etiology and pathology. Eur J Radiol 2011; 77: 228-234.

15. Murthy NS. The role of magnetic resonance imaging in scaphoid fractures. J Hand Surg 2013; 38: 2047-2054.

16. Donati OF, Zanetti M, Nagy L, et al. Is dynamic gadolinium enhancement needed in MR imaging for the preoperative assessment of scaphoidal viability in patients with scaphoid nonunion? Radiol 2011; 260: 808-816.

17. Magee T. Comparison of 3-T MRI and arthroscopy of intrinsic wrist ligament and TFCC tears. Am J Roentgenol 2009; 192: 80-85.

18. Hafezi-Nejad N, Carrino JA, Eng J, et al. Scapholunate interosseous ligament tears: diagnostic performance of 1.5 T, 3 T MRI, and MR arthrography - a systematic review and meta-analysis. Acad Radiol 2016; 23: 1091-1103.

19. Rüegger C, Schmid MR, Pfirrmann CW, et al. Peripheral tear of the triangular fibrocartilage: depiction with MR arthrography of the distal radioulnar joint. Am J Roentgenol 2007; 188: 187-192. 
20. DaSilva MF, Goodman AD, Gil JA, et al. Evaluation of ulnar-sided wrist pain. J Am Acad Orthop Surg 2017; 25: e150-e156.

21. Porteous R, Harish S, Parasu N. Imaging of ulnar-sided wrist pain. Can Assoc Radiol J 2012; 63: 18-29.

22. LiMarzi GM, O’Dell MC, Scherer K, et al. Magnetic resonance arthrography of the wrist and elbow. Magn Reson Imaging Clin 2015; 23: 441-455.

23. Schweitzer ME, Natale P, Winalski CS, et al. Indirect wrist MR arthrography: the effects of passive motion versus active exercise. Skeletal Radiol 2000; 29: 10-4.

24. Thomsen NO, Besjakov J, Björkman A. Accuracy of pre-and postcontrast, $3 \mathrm{~T}$ indirect $\mathrm{mr}$ arthrography compared with wrist arthroscopy in the diagnosis of wrist ligament injuries. J Wrist Surg 2018; 7: 382-388.

25. Dallaudière B, Meyer P, Larbi A, et al. Magnetic resonance arthrography of the wrist with axial traction: an iconographic review. Diagn Interv Imaging 2015; 96: 1307-1312.

26. Karantanas AH. Internal derangement of the hand and wrist. In: Taljanovic M, Omar IM, Hoover KB, et al. (ed.). Musculoskeletal Imaging. Oxford University Press, USA 2019.

27. Gupta P, Lenchik L, Wuertzer SD, et al. High-resolution 3-T MRI of the fingers: review of anatomy and common tendon and ligament injuries. Am J Roentgenol 2015; 204: W314-W323.

28. Parellada JA, Balkissoon AR, Hayes CW, et al. Bowstring injury of the flexor tendon pulley system: MR imaging. AJR Am J Roentgenol 1996; 167: 347-349.

29. Rawat U, Pierce JL, Evans S, et al. High-resolution MR imaging and US anatomy of the thumb. Radiographics 2016; 36: 1701-1716.

30. Kaewlai R, Avery LL, Asrani AV, et al. Multidetector CT of carpal injuries: anatomy, fractures, and fracture-dislocations. Radiographics 2008; 28: 1771-1784

31. Cerezal L, Piñal FD, Abascal F, et al. Imaging findings in ulnar-sided wrist impaction syndromes. Radiographics 2002; 22: 105-121.

32. Ng AW, Griffith JF, Fung CS, et al. MR imaging of the traumatic triangular fibrocartilaginous complex tear. Quant Imaging Med Surg 2017; 7: 443-460.

33. Burns JE, Tanaka T, Ueno T, et al. Pitfalls that may mimic injuries of the triangular fibrocartilage and proximal intrinsic wrist ligaments at $\mathrm{MR}$ imaging. Radiographics 2011; 31: 63-78.

34. Bateni CP, Bartolotta RJ, Richardson ML, et al. Imaging key wrist ligaments: what the surgeon needs the radiologist to know. Am J Roentgenol 2013; 200: 1089-1095.

35. Meyer P, Lintingre PF, Pesquer L, et al. The median nerve at the carpal tunnel... and elsewhere. J Belg Soc Radiol 2018; 102: 17.

36. Patel DB, Emmanuel NB, Stevanovic MV, et al. Hand infections: anatomy, types and spread of infection, imaging findings, and treatment options. Radiographics 2014; 34: 1968-1986.

37. Scheldrup EW. Tendon sheath patterns in the hand; an anatomical study based on 367 hand dissections. Surg Gynecol Obstet 1951; 93: 16-22.

38. Enhesari A, Saied A, Mohammadpoor L, et al. Presence or absence of palmaris longus and fifth superficial flexor digitorum; is there any effect on median nerve surface area in wrist sonography. Iran J Radiol 2014; 11: e14441.

39. Zeiss J, Jakab E, Khimji T, et al. The ulnar tunnel at the wrist (Guyon's canal): normal MR anatomy and variants. AJR Am J Roentgenol 1992; 158: 1081-1085.
40. Brown JM, Yablon CM, Morag Y, et al. US of the peripheral nerves of the upper extremity: a landmark approach. Radiographics 2016; 36 : 452-463.

41. Ali R, Khan F, Saeed S, et al. Lunotriquetral coalition: an unusual cause of wrist pain. Cureus 2019; 11: e5704.

42. Sookur PA, Naraghi AM, Bleakney RR, et al. Accessory muscles: anatomy, symptoms, and radiologic evaluation. Radiographics 2008; 28 : 481-99.

43. Rodríguez-Niedenführ M, Vázquez T, Golanó P, et al. Extensor digitorum brevis manus: anatomical, radiological and clinical relevance: a review. Clin Anat 2002; 15: 286-292.

44. Çavdar S, Doğan T, Bayramiçli M, et al. An unusual variation of extensor digitorum brevis manus: a case report and literature review. J Hand Surg 1998; 23: 173-177.

45. Propeck T, Quinn TJ, Jacobson JA, et al. Sonography and MR imaging of bifid median nerve with anatomic and histologic correlation. Am J Roentgenol 2000; 175: 1721-1725.

46. Hove LM. Epidemiology of scaphoid fractures in Bergen, Norway. Scand J Plast Reconstr Surg Hand Surg 1999; 33: 423-426.

47. Goldfarb CA, Yin Y, Gilula LA, et al. Wrist fractures: what the clinician wants to know. Radiology 2001; 219: 11-28.

48. Ng AW, Griffith JF, Taljanovic MS, et al. Is dynamic contrast-enhanced MRI useful for assessing proximal fragment vascularity in scaphoid fracture delayed and non-union? Skelet Radiol 2013; 42: 983-992.

49. Cerezal L, Abascal F, Canga A, et al. Usefulness of gadoliniumenhanced MR imaging in the evaluation of the vascularity of scaphoid nonunions. Am J Roentgenol 2000; 174: 141-149.

50. Donati OF, Zanetti M, Nagy L, et al. Is dynamic gadolinium enhancement needed in MR imaging for the preoperative assessment of scaphoidal viability in patients with scaphoid nonunion? Radiology 2011; 260: 808-816.

51. Raghupathi AK, Kumar P. Nonscaphoid carpal injuries - incidence and associated injuries. J Orthop 2014; 11: 91-95.

52. Squires JH, England E, Mehta K, et al. The role of imaging in diagnosing diseases of the distal radioulnar joint, triangular fibrocartilage complex, and distal ulna. Am J Roentgenol 2014; 203: 146-153.

53. Oneson SR, Scales LM, Timins ME, et al. MR imaging interpretation of the Palmer classification of triangular fibrocartilage complex lesions. Radiographics 1996; 16: 97-106.

54. Zanetti M, Hodler J, Gilula LA, et al. Assessment of dorsal or ventral intercalated segmental instability configurations of the wrist: reliability of sagittal MR images. Radiology 1998; 206: 339-345.

55. Taljanovic MS, Goldberg MR, Sheppard JE, et al. US of the intrinsic and extrinsic wrist ligaments and triangular fibrocartilage complex-normal anatomy and imaging technique. Radiographics 2011; 31: e44.

56. Ahmad T, Jan S, Rashid S. Management of flexor tendon injuries in hand. InTendons 2019. doi: 10.5772/intechopen.83483.

57. Plotkin B, Sampath SC, Sampath SC, et al. MR imaging and US of the wrist tendons. Radiographics 2016; 36: 1688-1700.

58. Lee RK, Griffith JF, Ng AW, et al. Cross-sectional area of the median nerve at the wrist: comparison of sonographic, MRI, and cadaveric measurements. J Clin Ultrasound 2019; 47: 122-127.

59. Ng AW, Griffith JF, Tong CS, et al. MRI criteria for diagnosis and predicting severity of carpal tunnel syndrome. Skeletal Radiol 2020; 49: 397-405. 
60. Park JS, Won HC, Oh JY, et al. Value of cross-sectional area of median nerve by MRI in carpal tunnel syndrome. Asian J Surg 2019; 43: 654-659.

61. Campagna R, Pessis E, Feydy A, et al. MRI assessment of recurrent carpal tunnel syndrome after open surgical release of the median nerve. Am J Roentgenol 2009; 193: 644-650.

62. Dong Q, Jacobson JA, Jamadar DA, et al. Entrapment neuropathies in the upper and lower limbs: anatomy and MRI features. Radiol Res Pract 2012; 2012: 230679.

63. Custer T, Channer LT, Hartranft TH. Hypothenar hammer syndrome: case report and literature review. Vasc Surg 1999; 33: 567-577.

64. Clavero JA, Alomar X, Monill JM, et al. MR imaging of ligament and tendon injuries of the fingers. Radiographics 2002; 22: 237-256.

65. Manaster BJ, Crim JR. Imaging anatomy: musculoskeletal e-book. Elsevier Health Sciences, 2016.

66. Clavero JA, Golanó P, Farinas O, et al. Extensor mechanism of the fingers: MR imaging-anatomic correlation. Radiographics 2003; 23: 593-611.

67. Kleinert HE. Primary repair of lacerated flexor tendons in "no man's land". J Bone Joint Surg Am 1967; 49: 577.

68. Verdan CE. Half a century of flexor tendon surgery. Current status and changing philosophies. Plast Reconstr Surg 1973; 51: 105-106.

69. Leddy JP, Packer JW. Avulsion of the profundus tendon insertion in athletes. J Hand Surg 1977; 2: 66-69.

70. Folmar RC, Nelson CL, Phalen GS. Ruptures of the flexor tendons in hands of non-rheumatoid patients. J Bone Joint Surg Am 1972; 54: 579-584.

71. Klauser A, Frauscher F, Bodner G, et al. Finger pulley injuries in extreme rock climbers: depiction with dynamic US. Radiology 2002; 222 755-761.

72. Susan S. Gray's Anatomy. The anatomical basis of clinical practice. Churchil Livingstone 2004.

73. Meraj S, Gyftopoulos S, Nellans K, et al. MRI of the extensor tendons of the wrist. Am J Roentgenol 2017; 209: 1093-1102.

74. Hirschmann A, Sutter R, Schweizer A, et al. MRI of the thumb: anatomy and spectrum of findings in asymptomatic volunteers. Am J Roentgenol 2014; 202: 819-827.

75. Rawat U, Pierce JL, Evans S, et al. High-resolution MR imaging and US anatomy of the thumb. Radiographics 2016; 36: 1701-1716.

76. Melville DM, Taljanovic MS, Scalcione LR, et al. Imaging and management of thumb carpometacarpal joint osteoarthritis. Skeletal Radiol 2015; 44: 165-177.

77. Baskies MA, Lee SK. Evaluation and treatment of injuries of the ulnar collateral ligament of the thumb metacarpophalangeal joint. Bull NYU Hosp Jt Dis 2009; 67: 68-74.

78. Plancher KD, Ho CP, Cofield SS, et al. Role of MR imaging in the management of "skier's thumb" injuries. Magn Reson Imaging Clin N Am 1999; 7: 73-84.

79. Harper MT, Chandnani VP, Spaeth J, et al. Gamekeeper thumb: diagnosis of ulnar collateral ligament injury using magnetic resonance imaging, magnetic resonance arthrography and stress radiography. J Magn Reson Imaging 1996; 6: 322-328.

80. Gluck JS, Balutis EC, Glickel SZ. Thumb ligament injuries. J Hand Surg 2015; 40: 835-842.

81. Doty JF, Rudd JN, Jemison M. Radial collateral ligament injury of the thumb with a Stener-like lesion. Orthopedics 2010; 33: 925. 